\title{
Error bounds for augmented truncations of discrete-time block-monotone Markov chains under subgeometric drift conditions*
}

\author{
Hiroyuki Masuyamał \\ Department of Systems Science, Graduate School of Informatics, Kyoto University \\ Kyoto 606-8501, Japan
}

\begin{abstract}
This paper studies the last-column-block-augmented northwest-corner truncation (LCblock-augmented truncation, for short) of discrete-time block-monotone Markov chains under subgeometric drift conditions. The main result of this paper is to present an upper bound for the total variation distance between the stationary probability vectors of a block-monotone Markov chain and its LC-block-augmented truncation. The main result is extended to Markov chains that themselves may not be block monotone but are block-wise dominated by block-monotone Markov chains satisfying modified drift conditions. Finally, as an application of the obtained results, the GI/G/1-type Markov chain is considered.
\end{abstract}

Keywords: Last-column-block-augmented (LC-block-augmented), northwest-corner truncation, block monotonicity, subgeometric drift condition, GI/G/1-type Markov chain

Mathematics Subject Classification: 60J10, 60J22, 60K25

\section{Introduction}

This paper considers the truncation approximation of discrete-time block-structured Markov chains characterized by an infinite number of block matrices. The typical examples of such Markov chains are M/G/1-, GI/M/1- and GI/G/1-type Markov chains and level-dependent quasibirth-and-death processes (LD-QBDs) (see, e.g., [6] and the references therein) and they appear as the queue length processes of various semi-Markovian queues (see, e.g., [23]).

It is a challenging problem to obtain the stationary probability vectors of block-structured Markov chains characterized by an infinite number of block matrices. To solve this problem, we often use the augmented northwest-corner truncation (augmented truncation, for short). More specifically, we form a finite stochastic matrix by augmenting, in some way, the (finite) northwest-corner truncation of the transition probability matrix and then adopt the stationary

${ }^{*}$ This paper is published in SIAM Journal on Matrix Analysis and Applications, vol. 37, no. 3, pp. 877-910, 2016.

†E-mail: masuyama@sys.i.kyoto-u.ac.jp 
probability vector of the resulting finite stochastic matrix as an approximation to that of the original Markov chain.

Although there are infinitely many variations of such augmented truncation, this paper focuses on the last-column-block-augmented northwest-corner truncation (LC-block-augmented truncation, for short) because it is proved that the LC-block-augmentation is the best (in a certain sense) among all the block augmentations if they are applied to the northwest-corner truncations of block-monotone transition probability matrices (see [10, Theorem 3.6] and [20, Theorem 4.1]). Note that block monotonicity is an extension of (classical) monotonicity to block-structured Markov chains [10, Definition 2.5].

The main purpose of this paper is to estimate the error of the stationary probability vector obtained by the LC-block-augmented truncation. There are some related studies on the truncation of Markov chains. Tweedie [24] presented a total-variation-distance error bound for the stationary probability vector of the last-column-augmented northwest-corner truncation of a monotone and geometrically ergodic Markov chain in discrete time. More precisely, Tweedie [24]'s bound is an upper bound for the total variation distance between the stationary probability vectors of the original Markov chain and its last-column-augmented northwest-corner truncation. Hart and Tweedie [5] and Liu [13] discussed the convergence of the stationary probability vectors of the augmented truncations of continuous-time Markov chains with monotonicity and/or exponential ergodicity. Masuyama [18, 20] extended Tweedie [24]'s result to block-monotone Markov chains in discrete and continuous time.

Without the monotonicity of Markov chains, Hervé and Ledoux [7] derived a total-variationdistance error bound for the stationary probability vector of the last-column-augmented northwestcorner truncation of a geometrically ergodic Markov chain in discrete time. Zeifman et al. [27] considered a periodic and exponentially weakly ergodic non-time-homogeneous birth-and-death process in continuous time, and they presented a total-variation-distance error bound for the periodic stationary distribution obtained by the truncation of the state space (see also [25, 26]).

Basically, all the existing results mentioned above assume geometric ergodicity (exponential ergodicity in continuous time) and thus they are not applicable to Markov chains with subgeometric ergodicity (including polynomial ergodicity). For example, reflected Markov additive processes and GI/G/1-type Markov chains with the heavy-tailed asymptotics [8, 14], which typically arise from BMAP/GI/1 queues with subexponential service times and/or batch sizes [15, 19]. As far as we know, there are no studies on the error estimation of the augmented truncation of Markov chains with subgeometric ergodicity, except for Liu [12]'s work. Liu [12] derived a total-variation-distance error bound for the stationary probability vector of the last-column-augmented northwest-corner truncation of a monotone and polynomially ergodic Markov chain in discrete time.

In this paper, we consider a block-monotone Markov chain under the drift condition proposed by Douc et al. [3], which covers polynomial ergodicity and other types of subgeometric ergodicity. We first derive an upper bound for the total variation distance between the stationary probability vectors of the original Markov chain and its LC-block-augmented truncation, which is the main result of this paper. We also present a similar bound in the case where 
the original Markov chain itself may not be block monotone but is block-wise dominated by a block-monotone Markov chain satisfying a modified drift condition with a larger tolerance for boundary exceptions. The modified drift condition strengthens the applicability of the obtained bound. Finally, we provide a detailed procedure for establishing our error bounds for the LC-block-augmented truncations of block-monotone GI/G/1-type Markov chains.

The rest of this paper is divided into five sections. Section 2 provides preliminary results on block-monotone stochastic matrices. Section 3 presents the main result of this paper. Section 4 contains the extension of the main result to possibly non-block-monotone Markov chains that are block-wise dominated by block-monotone Markov chains satisfying the modified drift condition. Section 5 considers the application of the extended result to block-monotone GI/G/1type Markov chains. Finally, Section 6 provides concluding remarks.

\section{Preliminaries}

Let $\left\{\left(X_{\nu}, J_{\nu}\right) ; \nu \in \mathbb{Z}_{+}\right\}$denote a Markov chain with state space $\mathbb{F}=\mathbb{Z}_{+} \times \mathbb{D}$, where $\mathbb{Z}_{+}=$ $\{0,1,2, \ldots\}$ and $\mathbb{D}=\{1,2, \ldots, d\} \subset \mathbb{N}:=\{1,2, \ldots\}$. Let $\boldsymbol{P}:=(p(k, i ; \ell, j))_{(k, i),(\ell, j) \in \mathbb{F}}$ denote the transition probability matrix of the Markov chain $\left\{\left(X_{\nu}, J_{\nu}\right)\right\}$, i.e.,

$$
p(k, i ; \ell, j)=\mathrm{P}\left(X_{\nu+1}=\ell, J_{\nu+1}=j \mid X_{\nu}=k, J_{\nu}=i\right), \quad(k, i ; \ell, j) \in \mathbb{F}^{2},
$$

where $(k, i ; \ell, j)$ represents ordered pair $((k, i),(\ell, j))$. Note here that $\boldsymbol{P}$ is row stochastic (stochastic, for short, hereafter), i.e., $P e=e$, where $e$ denotes a column vector of 1 's of an appropriate order.

For $n \in \mathbb{N}$, let $\left.{ }_{(n)} \boldsymbol{P}_{n}:={ }_{\left({ }_{(n)}\right.} p_{n}(k, i ; \ell, j)\right)_{(k, i),(\ell, j) \in \mathbb{F}}$ denote a stochastic matrix such that, for $i, j \in \mathbb{D}$,

$$
{ }_{(n)} p_{n}(k, i ; \ell, j)= \begin{cases}p(k, i ; \ell, j), & k \in \mathbb{Z}_{+}, \ell=0,1, \ldots, n-1, \\ \sum_{m=n}^{\infty} p(k, i ; m, j), & k \in \mathbb{Z}_{+}, \ell=n, \\ 0, & \text { otherwise. }\end{cases}
$$

By definition, $\boldsymbol{P}$ and ${ }_{(n)} \boldsymbol{P}_{n}$ can be partitioned into block matrices with size $d$. Furthermore, (2.1) implies that ${ }_{(n)} \boldsymbol{P}_{n}$ is in the following form:

$$
\begin{aligned}
& \mathbb{F} \leqslant n \quad \mathbb{F} \backslash \mathbb{F} \leqslant n \\
& { }_{(n)} \boldsymbol{P}_{n}=\begin{array}{l}
\mathbb{F}^{\leqslant n} \\
\mathbb{F} \backslash \mathbb{F} \leqslant n
\end{array}\left(\begin{array}{cc}
{ }_{(n)} \boldsymbol{P}_{n}^{\leqslant n} & \boldsymbol{O} \\
{ }_{(n)} \boldsymbol{P}_{n}^{*} & \boldsymbol{O}
\end{array}\right),
\end{aligned}
$$

where $\mathbb{F}^{\leqslant n}=\{0,1, \ldots, n\} \times \mathbb{D}$ and $\boldsymbol{O}$ denotes the zero matrix of an appropriate order. Equation (2.2) shows that the sub-state space $\mathbb{F} \backslash \mathbb{F}^{\leqslant n}$ of ${ }_{(n)} \boldsymbol{P}_{n}$ is transient and thus the submatrix ${ }_{(n)} \boldsymbol{P}_{n}^{*}$ of ${ }_{(n)} \boldsymbol{P}_{n}$ does not have any contribution to the stationary probability vector of ${ }_{(n)} \boldsymbol{P}_{n}$. Therefore, we call the whole matrix ${ }_{(n)} \boldsymbol{P}_{n}$ the last-column-block-augmented (LC-block-augmented) 
northwest-corner truncation (LC-block-augmented truncation, for short). The LC-block-augmented truncation ${ }_{(n)} \boldsymbol{P}_{n}$ is also called the last-column-block-augmented first-n-block-column truncation in [18]. It should be noted that, since ${ }_{(n)} \boldsymbol{P}_{n}^{\leqslant n}$ in (2.2) is a finite stochastic matrix, the LC-block-augmented truncation ${ }_{(n)} \boldsymbol{P}_{n}$ always has at least one stationary probability vector (see, e.g., [2, Chapter 3, Theorems 3.1 and 3.3]), which is denoted by ${ }_{(n)} \boldsymbol{\pi}_{n}:=\left({ }_{(n)} \pi_{n}(k, i)\right)_{(k, i) \in \mathbb{F}}$.

We now assume that $\boldsymbol{P}$ is irreducible and positive recurrent. We then define $\boldsymbol{\pi}:=(\pi(k, i))_{(k, i) \in \mathbb{F}}$ as the unique stationary probability vector of $\boldsymbol{P}$. We also assume, unless otherwise stated, that $\boldsymbol{P}$ is block monotone with block sized $d$ (see [18, Definition 1.1]), i.e.,

$$
\sum_{m=\ell}^{\infty} \boldsymbol{P}(k ; m) \leq \sum_{m=\ell}^{\infty} \boldsymbol{P}(k+1 ; m), \quad k, \ell \in \mathbb{Z}_{+},
$$

where $\boldsymbol{P}(k ; \ell):=(p(k, i ; \ell, j))_{i, j \in \mathbb{D}}$ is the $(k, \ell)$ th block of $\boldsymbol{P}$. To shorten the statements on block monotonicity, let $\mathrm{BM}_{d}$ denote the set of block-monotone stochastic matrices with block size $d$. It then follows from $\boldsymbol{P} \in \mathrm{BM}_{d}$ that a stochastic matrix $\sum_{m=0}^{\infty} \boldsymbol{P}(k ; m)$ is constant with $k \in \mathbb{Z}_{+}$(see [18, Proposition 1.1]). Furthermore, since $\boldsymbol{P}$ is irreducible, the stochastic matrix $\boldsymbol{\Psi}:=\sum_{m=0}^{\infty} \boldsymbol{P}(k ; m)$ is irreducible and thus has the unique stationary probability vector, denoted by $\varpi:=(\varpi(i))_{i \in \mathbb{D}}$.

Finally, we introduce some symbols and definitions related to block monotonicity. Let

$$
\boldsymbol{T}_{d}=\left(\begin{array}{ccccc}
\boldsymbol{I}_{d} & \boldsymbol{O} & \boldsymbol{O} & \boldsymbol{O} & \ldots \\
\boldsymbol{I}_{d} & \boldsymbol{I}_{d} & \boldsymbol{O} & \boldsymbol{O} & \ldots \\
\boldsymbol{I}_{d} & \boldsymbol{I}_{d} & \boldsymbol{I}_{d} & \boldsymbol{O} & \ldots \\
\boldsymbol{I}_{d} & \boldsymbol{I}_{d} & \boldsymbol{I}_{d} & \boldsymbol{I}_{d} & \ldots \\
\vdots & \vdots & \vdots & \vdots & \ddots
\end{array}\right), \boldsymbol{T}_{d}^{-1}=\left(\begin{array}{ccccc}
\boldsymbol{I}_{d} & \boldsymbol{O} & \boldsymbol{O} & \boldsymbol{O} & \ldots \\
-\boldsymbol{I}_{d} & \boldsymbol{I}_{d} & \boldsymbol{O} & \boldsymbol{O} & \ldots \\
\boldsymbol{O} & -\boldsymbol{I}_{d} & \boldsymbol{I}_{d} & \boldsymbol{O} & \ldots \\
\boldsymbol{O} & \boldsymbol{O} & -\boldsymbol{I}_{d} & \boldsymbol{I}_{d} & \ldots \\
\vdots & \vdots & \vdots & \vdots & \ddots
\end{array}\right)
$$

where $\boldsymbol{I}_{d}$ denotes the $d \times d$ identity matrix (we write $\boldsymbol{I}$ for the identity matrix whose order is clear from the context). Note here that (2.3) is equivalent to

$$
\boldsymbol{T}_{d}^{-1} \boldsymbol{P} \boldsymbol{T}_{d} \geq \boldsymbol{O}
$$

Definition 2.1 A column vector $\boldsymbol{f}=(f(k, i))_{(k, i) \in \mathbb{F}}$ with block size $d$ is said to be block increasing if $\boldsymbol{T}_{d}^{-1} \boldsymbol{f} \geq \mathbf{0}$, i.e., $f(k, i) \leq f(k+1, i)$ for all $(k, i) \in \mathbb{Z}_{+} \times \mathbb{D}$. We denote by $\mathrm{BI}_{d}$ the set of block-increasing column vectors with block size $d$.

Definition 2.2 A probability vector $\boldsymbol{\mu}:=(\mu(k, i))_{(k, i) \in \mathbb{F}}$ with block size $d$ is said to be blockwise dominated by a probability vector $\boldsymbol{\eta}:=(\eta(k, i))_{(k, i) \in \mathbb{F}}$ (denoted by $\left.\boldsymbol{\mu} \prec_{d} \boldsymbol{\eta}\right)$ if $\boldsymbol{\mu} \boldsymbol{T}_{d} \leq$ $\boldsymbol{\eta} \boldsymbol{T}_{d}$. Similarly, a stochastic matrix $\boldsymbol{P}_{1}:=\left(p_{1}(k, i ; \ell, j)\right)_{(k, i),(\ell, j) \in \mathbb{F}}$ with block size $d$ is said to be block-wise dominated by a stochastic matrix $\boldsymbol{P}_{2}:=\left(p_{2}(k, i ; \ell, j)\right)_{(k, i),(\ell, j) \in \mathbb{F}}$ (denoted by $\left.\boldsymbol{P}_{1} \prec{ }_{d} \boldsymbol{P}_{2}\right)$ if $\boldsymbol{P}_{1} \boldsymbol{T}_{d} \leq \boldsymbol{P}_{2} \boldsymbol{T}_{d}$. 
It is known that if $\boldsymbol{P} \in \mathrm{BM}_{d}$ then ${ }_{(n)} \boldsymbol{P}_{n} \prec_{d} \boldsymbol{P}$ and thus ${ }_{(n)} \boldsymbol{\pi}_{n} \prec_{d} \boldsymbol{\pi}$ (see [18, Proposition 2.3]), which leads to

$$
\sum_{k=0}^{\infty}{ }_{(n)} \pi_{n}(k, i)=\sum_{k=0}^{\infty} \pi(k, i)=\varpi(i), \quad i \in \mathbb{D}
$$

\section{Main result}

This section presents an upper bound for $\left\|_{(n)} \boldsymbol{\pi}_{n}-\boldsymbol{\pi}\right\|$, where $\|\cdot\|$ denotes the total variation distance, i.e.,

$$
\left\|_{(n)} \boldsymbol{\pi}_{n}-\boldsymbol{\pi}\right\|=\left.\sum_{(k, i) \in \mathbb{F}}\right|_{(n)} \pi_{n}(k, i)-\pi(k, i) \mid .
$$

Let $\boldsymbol{p}^{m}(k, i):=\left(p^{m}(k, i ; \ell, j)\right)_{(\ell, j) \in \mathbb{F}}$ and ${ }_{(n)} \boldsymbol{p}_{n}^{m}(k, i):=\left({ }_{(n)} p_{n}^{m}(k, i ; \ell, j)\right)_{(\ell, j) \in \mathbb{F}}$ denote probability vectors such that $p^{m}(k, i ; \ell, j)$ and ${ }_{(n)} p_{n}^{m}(k, i ; \ell, j)$ represent the $(k, i ; \ell, j)$ th elements of $\boldsymbol{P}^{m}$ and $\left.{ }_{\left({ }_{(n)}\right.} \boldsymbol{P}_{n}\right)^{m}$, respectively. For any function $\varphi(\cdot, \cdot)$ on $\mathbb{F}$, let $\varphi(k, \varpi)=\sum_{i \in \mathbb{D}} \varpi(i) \varphi(k, i)$ for $k \in \mathbb{Z}_{+}$. We then have

$$
\begin{gathered}
\left\|_{(n)} \boldsymbol{\pi}_{n}-\boldsymbol{\pi}\right\| \leq\left\|\boldsymbol{p}^{m}(0, \varpi)-\boldsymbol{\pi}\right\|+\left\|_{(n)} \boldsymbol{p}_{n}^{m}(0, \varpi)-{ }_{(n)} \boldsymbol{\pi}_{n}\right\| \\
+\left\|_{(n)} \boldsymbol{p}_{n}^{m}(0, \varpi)-\boldsymbol{p}^{m}(0, \varpi)\right\|
\end{gathered}
$$

It also follows from the second last inequality in the proof of [18, Theorem 3.1] that

$$
\left\|_{(n)} \boldsymbol{p}_{n}^{m}(0, \varpi)-\boldsymbol{p}^{m}(0, \varpi)\right\| \leq 2 m \sum_{i \in \mathbb{D}}(n) \pi_{n}(n, i)
$$

To estimate the first and second terms on the right hand side of (3.1), we assume the subgeometric drift condition proposed in [3], which is described in Assumption 3.1 below. For the description of the drift condition, let $\mathbf{1}_{K}=\left(1_{K}(k, i)\right)_{(k, i) \in \mathbb{F}}, K \in \mathbb{Z}_{+}$denote a column vector such that

$$
1_{K}(k, i)= \begin{cases}1, & (k, i) \in \mathbb{F} \leqslant K \\ 0, & (k, i) \in \mathbb{F} \backslash \mathbb{F} \leqslant K\end{cases}
$$

In addition, for any scalar-valued function $\theta$ on $(-\infty, \infty)$ and any real-valued column vector $\boldsymbol{a}:=(a(i))$, let $\theta \circ \boldsymbol{a}=(\theta \circ a(i))$.

Assumption 3.1 ([33, Condition $\mathbf{D}(\phi, V, C)])$ There exist a constant $b \in(0, \infty)$, a column vector $\boldsymbol{v}=(v(k, i))_{(k, i) \in \mathbb{F}} \in \mathrm{BI}_{d}$ with $\boldsymbol{v} \geq \boldsymbol{e}$, and a nondecreasing differentiable concave function $\phi:[1, \infty) \rightarrow(0, \infty)$ with $\lim _{t \rightarrow \infty} \phi^{\prime}(t)=0$ such that

$$
\boldsymbol{P} \boldsymbol{v} \leq \boldsymbol{v}-\phi \circ \boldsymbol{v}+b \mathbf{1}_{0}
$$


Remark 3.1 If $\lim _{t \rightarrow \infty} \phi^{\prime}(t)=c$ for some $c>0$, then Assumption 3.1 is reduced to the geometric drift condition (see [3, Remark 1] for the details): There exist $b \in(0, \infty), \gamma \in(0,1)$ and a column vector $\boldsymbol{v} \in \mathrm{BI}_{d}$ with $v \geq e$ such that

$$
\boldsymbol{P} \boldsymbol{v} \leq \gamma \boldsymbol{v}+b \mathbf{1}_{0}
$$

which is assumed in the related studies [7, 18, 24].

Under Assumption 3.1, the irreducible stochastic matrix $\boldsymbol{P}$ is subgeometrically ergodic if $\boldsymbol{P}$ is aperiodic [3, Proposition 2.5]. However, we do not necessarily assume the aperiodicity of $P$.

We now introduce some symbols according to [3, Section 2]. Let $H_{\phi}$ denote a function on $[1, \infty)$ such that

$$
H_{\phi}(x)=\int_{1}^{x} \frac{\mathrm{d} y}{\phi(y)}, \quad x \geq 1 .
$$

Clearly, $H_{\phi}$ is an increasing differentiable concave function, and $\lim _{x \rightarrow \infty} H_{\phi}(x)=\infty$ due to the concavity of $\phi$ (see [3, section 2]). Thus, the inverse $H_{\phi}^{-1}:[0, \infty) \rightarrow[1, \infty)$ of $H_{\phi}$ is welldefined and $\lim _{x \rightarrow \infty} H_{\phi}^{-1}(x)=\infty$. Furthermore, it follows from (3.4) that $H_{\phi}^{-1}$ is an increasing differentiable function and

$$
r_{\phi}(x):=\left(H_{\phi}^{-1}\right)^{\prime}(x)=\phi \circ H_{\phi}^{-1}(x), \quad x \geq 0 .
$$

The function $r_{\phi}$ is nondecreasing because $\phi$ is nondecreasing and $H_{\phi}^{-1}$ is increasing. In addition, it follows from [3, Proposition 2.1] that $r_{\phi}$ is log-concave. For convenience, we define $r_{\phi}(x)=0$ for $x<0$.

In what follows, we present two lemmas and the main result of this paper.

Lemma 3.1 Consider the Markov chain $\left\{\left(X_{\nu}, J_{\nu}\right) ; \nu \in \mathbb{Z}_{+}\right\}$with state space $\mathbb{F}$ and transition probability matrix $\boldsymbol{P}$, and let $\tau_{0}^{+}=\inf \left\{\nu \in \mathbb{N} ; X_{\nu}=0\right\}$. If $\boldsymbol{P} \in \mathrm{BM}_{d}, \boldsymbol{P}$ is irreducible and Assumption 3.1 holds, then

$$
\begin{aligned}
\mathrm{E}_{(k, i)}\left[r_{\phi}\left(\tau_{0}^{+}-1\right)\right] & \leq v(k \vee 1, i), \quad(k, i) \in \mathbb{F}, \\
\mathrm{E}_{\boldsymbol{\pi}}\left[r_{\phi}\left(\tau_{0}^{+}-1\right)\right] & \leq v(k \vee 1, \varpi), \quad k \in \mathbb{Z}_{+},
\end{aligned}
$$

where $x \vee y=\max (x, y)$ and

$$
\begin{aligned}
\mathrm{E}_{\boldsymbol{\pi}}[\cdot] & =\sum_{(k, i) \in \mathbb{F}} \pi(k, i) \mathrm{E}_{(k, i)}[\cdot], \\
\mathrm{E}_{(k, i)}[\cdot] & =\mathrm{E}\left[\cdot \mid X_{0}=k, J_{0}=i\right], \quad(k, i) \in \mathbb{F} .
\end{aligned}
$$

Proof. We first prove (3.6). Note that

$$
\mathrm{E}_{(k, i)}\left[r_{\phi}\left(\tau_{0}^{+}-1\right)\right] \leq \mathrm{E}_{(k, i)}\left[\sum_{n=0}^{\tau_{0}^{+}-1} r_{\phi}(n)\right], \quad(k, i) \in \mathbb{F} .
$$


It then follows from [3, Proposition 2.2] that

$$
\mathrm{E}_{(k, i)}\left[\sum_{n=0}^{\tau_{0}^{+}-1} r_{\phi}(n)\right] \leq v(k, i), \quad k \in \mathbb{N}, i \in \mathbb{D} .
$$

Furthermore,

$$
\mathrm{E}_{(0, i)}\left[\sum_{n=0}^{\tau_{0}^{+}-1} r_{\phi}(n)\right] \leq \mathrm{E}_{(k, i)}\left[\sum_{n=0}^{\tau_{0}^{+}-1} r_{\phi}(n)\right], \quad k \in \mathbb{N}, i \in \mathbb{D},
$$

which follows from the pathwise-ordered property [18, Lemma A.1] of the block-monotone Markov chain $\left\{\left(X_{\nu}, J_{\nu}\right)\right\}$. Combining (3.9) and (3.10), we have

$$
\mathrm{E}_{(k, i)}\left[\sum_{n=0}^{\tau_{0}^{+}-1} r_{\phi}(n)\right] \leq v(k \vee 1, i), \quad(k, i) \in \mathbb{F} .
$$

Substituting (3.11) into (3.8), we obtain (3.6).

Next, we prove (3.7). It follows from [22, Theorem 10.4.9] that, for any function $\varphi(\cdot, \cdot)$ on $\mathbb{F}$ such that $\sum_{(k, i) \in \mathbb{F}} \pi(k, i)|\varphi(k, i)|<\infty$,

$$
\sum_{(k, i) \in \mathbb{F}} \pi(k, i) \varphi(k, i)=\sum_{i \in \mathbb{D}} \pi(0, i) \mathrm{E}_{(0, i)}\left[\sum_{\nu=0}^{\tau_{0}^{+}-1} \varphi\left(X_{\nu}, J_{\nu}\right)\right] .
$$

Note here that $\mathrm{E}_{\pi}\left[r_{\phi}\left(\tau_{0}^{+}-1\right)\right]=\mathrm{E}_{\pi}\left[\sum_{n=0}^{\tau_{0}^{+}-1} \Delta r_{\phi}(n)\right]$, where $\Delta r_{\phi}(n)=r_{\phi}(n)-r_{\phi}(n-1)$ for $n \in \mathbb{Z}_{+}$. Thus, letting $\varphi(k, i)=\mathrm{E}_{(k, i)}\left[\sum_{n=0}^{\tau_{0}^{+}-1} \Delta r_{\phi}(n)\right]$ in (3.12), we obtain

$$
\begin{aligned}
\mathrm{E}_{\boldsymbol{\pi}}\left[r_{\phi}\left(\tau_{0}^{+}-1\right)\right] & =\mathrm{E}_{\boldsymbol{\pi}}\left[\sum_{n=0}^{\tau_{0}^{+}-1} \Delta r_{\phi}(n)\right] \\
& =\sum_{i \in \mathbb{D}} \pi(0, i) \mathrm{E}_{(0, i)}\left[\sum_{\nu=0}^{\tau_{0}^{+}-1} \mathrm{E}_{\left(X_{\nu}, J_{\nu}\right)}\left[\sum_{n=\nu}^{\tau_{0}^{+}(\nu)-1} \Delta r_{\phi}(n)\right]\right],
\end{aligned}
$$

where $\tau_{0}^{+}(\nu)=\inf \left\{n \geq \nu+1 ; X_{n}=0\right\}$. Changing the order of summation on the right hand side of (3.13), we have

$$
\begin{aligned}
\mathrm{E}_{\boldsymbol{\pi}} & {\left[r_{\phi}\left(\tau_{0}^{+}-1\right)\right] } \\
& =\sum_{i \in \mathbb{D}} \pi(0, i) \mathrm{E}_{(0, i)}\left[\sum_{n=0}^{\tau_{0}^{+}-1} \sum_{\nu=0}^{n} \Delta r_{\phi}(n)\right] \\
& =\sum_{i \in \mathbb{D}} \pi(0, i) \mathrm{E}_{(0, i)}\left[\sum_{n=0}^{\tau_{0}^{+}-1} r_{\phi}(n)\right] \leq \sum_{i \in \mathbb{D}} \varpi(i) \mathrm{E}_{(k, i)}\left[\sum_{n=0}^{\tau_{0}^{+}-1} r_{\phi}(n)\right], \quad k \in \mathbb{Z}_{+},
\end{aligned}
$$


where the last inequality follows from (3.10) and $\pi(0, i) \leq \varpi(i)$ for $i \in \mathbb{D}$ (see (2.4)). Applying (3.11) to (3.14) yields (3.7).

Using Lemma 3.1, we prove the following lemma.

Lemma 3.2 Suppose that $\boldsymbol{P} \in \mathrm{BM}_{d}$ and $\boldsymbol{P}$ is irreducible. If Assumption 3.1 holds, then, for all $k \in \mathbb{Z}_{+}$and $m \in \mathbb{N}$,

$$
\begin{aligned}
\left\|\boldsymbol{p}^{m}(k, \varpi)-\boldsymbol{\pi}\right\| & \leq \frac{4}{r_{\phi}(m-1)} v(k \vee 1, \varpi), \\
\left\|_{(n)} \boldsymbol{p}_{n}^{m}(k, \varpi)-{ }_{(n)} \boldsymbol{\pi}_{n}\right\| & \leq \frac{4}{r_{\phi}(m-1)} v(k \vee 1, \varpi), \quad n \in \mathbb{N},
\end{aligned}
$$

where the function $r_{\phi}$ is given in (3.5).

Proof. We first prove (3.15). Let $\tau_{0}=\inf \left\{n \in \mathbb{Z}_{+} ; X_{n}=0\right\}$ and $\mathrm{P}_{(k, i)}(\cdot)=\mathrm{P}\left(\cdot \mid X_{0}=\right.$ $\left.k, J_{0}=i\right)$ for $(k, i) \in \mathbb{F}$. Following the derivation of [18, Eq. (3.22)] and replacing $\boldsymbol{v}$ by $\boldsymbol{e}$, we have

$$
\begin{aligned}
\left\|\boldsymbol{p}^{m}(k, \varpi)-\boldsymbol{\pi}\right\| & \leq 2 \sum_{i \in \mathbb{D}} \varpi(i) \mathrm{P}_{(k, i)}\left(\tau_{0}>m\right)+2 \sum_{(\ell, j) \in \mathbb{F}} \pi(\ell, j) \mathrm{P}_{(\ell, j)}\left(\tau_{0}>m\right) \\
& \leq 2 \sum_{i \in \mathbb{D}} \varpi(i) \mathrm{P}_{(k, i)}\left(\tau_{0}^{+}>m\right)+2 \sum_{(\ell, j) \in \mathbb{F}} \pi(\ell, j) \mathrm{P}_{(\ell, j)}\left(\tau_{0}^{+}>m\right),
\end{aligned}
$$

where the second inequality follows from the fact that $\mathrm{P}_{(\ell, j)}\left(\tau_{0}>m\right) \leq \mathrm{P}_{(\ell, j)}\left(\tau_{0}^{+}>m\right)$ for $m \in \mathbb{N}$ and $(\ell, j) \in \mathbb{F}$. Using Markov's inequality, we have

$$
\mathrm{P}_{(\ell, j)}\left(\tau_{0}^{+}>m\right) \leq \frac{1}{r_{\phi}(m-1)} \mathrm{E}_{(\ell, j)}\left[r_{\phi}\left(\tau_{0}^{+}-1\right)\right], \quad m \in \mathbb{N},(\ell, j) \in \mathbb{F} .
$$

Thus

$$
\left\|\boldsymbol{p}^{m}(k, \varpi)-\boldsymbol{\pi}\right\| \leq \frac{2}{r_{\phi}(m-1)}\left(\sum_{i \in \mathbb{D}} \varpi(i) \mathrm{E}_{(k, i)}\left[r_{\phi}\left(\tau_{0}^{+}-1\right)\right]+\mathrm{E}_{\boldsymbol{\pi}}\left[r_{\phi}\left(\tau_{0}^{+}-1\right)\right\}(3.18)\right.
$$

Applying Lemma 3.1 to (3.18) yields (3.15). In addition, since ${ }_{(n)} \boldsymbol{P}_{n} \prec_{d} \boldsymbol{P}$ and $\boldsymbol{v} \in \mathrm{BI}_{d}$, it follows from (3.3) and [18, Remark 2.1] that

$$
{ }_{(n)} \boldsymbol{P}_{n} \boldsymbol{v} \leq \boldsymbol{P} \boldsymbol{v} \leq \boldsymbol{v}-\phi \circ \boldsymbol{v}+b \mathbf{1}_{0}
$$

Therefore, (3.16) can be proved in the same way as that of (3.15). The details are omitted.

Theorem 3.1 below presents upper bounds for $\left\|_{(n)} \boldsymbol{\pi}_{n}-\boldsymbol{\pi}\right\|$, which are the main result of this paper. 
Theorem 3.1 Suppose that $\boldsymbol{P} \in \mathrm{BM}_{d}$ and $\boldsymbol{P}$ is irreducible. If Assumption 3.1 holds, then, for all $n, m \in \mathbb{N}$,

$$
\begin{aligned}
& \left\|_{(n)} \boldsymbol{\pi}_{n}-\boldsymbol{\pi}\right\| \leq \frac{8}{r_{\phi}(m-1)} v(1, \varpi)+2 m \sum_{i \in \mathbb{D}}(n) \pi_{n}(n, i), \\
& \left\|_{(n)} \boldsymbol{\pi}_{n}-\boldsymbol{\pi}\right\| \leq \frac{8}{r_{\phi}(m-1)} v(1, \varpi)+2 m b \sum_{i \in \mathbb{D}} \frac{1}{\phi \circ v(n, i)},
\end{aligned}
$$

where the function $r_{\phi}$ is given in (3.5).

Proof. Substituting (3.2), (3.15) and (3.16) into (3.1), we obtain (3.20). Furthermore, premultiplying both sides of (3.19) by ${ }_{(n)} \boldsymbol{\pi}_{n}$, we have

$$
\sum_{(k, i) \in \mathbb{F}}(n) \pi_{n}(k, i) \cdot \phi \circ v(k, i) \leq b,
$$

which leads to

$$
{ }_{(n)} \pi_{n}(n, i) \leq \frac{b}{\phi \circ v(n, i)}
$$

Combining (3.20) and (3.22) yields (3.21).

Remark 3.2 Suppose that $\lim _{t \rightarrow \infty} \phi(t)=\infty$ and $\lim _{k \rightarrow \infty} v(k, i)=\infty$ for all $i \in \mathbb{D}$. It then holds that $\lim _{n \rightarrow \infty} \phi \circ v(n, i)=\infty$ for $i \in \mathbb{D}$. Thus, it follows from (3.22) that $\lim _{n \rightarrow \infty}(n) \pi_{n}(n, i)=$ 0 for $i \in \mathbb{D}$. Recall here that $\lim _{x \rightarrow \infty} H_{\phi}^{-1}(x)=\infty$. Applying this and $\lim _{t \rightarrow \infty} \phi(t)=\infty$ to (3.5) yields $\lim _{x \rightarrow \infty} r_{\phi}(x)=\infty$. Therefore, we can reduce the error bounds (3.20) and (3.21) to any desired value less than two by fixing $m \in \mathbb{N}$ sufficient large and then choosing $n \in \mathbb{N}$ large such that the bounds take the desired value.

\section{Extension of the main result}

In this section, we extend Theorem 3.1 to the case where $\boldsymbol{P}$ may not be block monotone but is block-wise dominated by a block-monotone stochastic matrix $\widetilde{\boldsymbol{P}}=(\widetilde{p}(k, i ; \ell, j))_{(k, i),(\ell, j) \in \mathbb{F}}$. Note here that $\widetilde{\boldsymbol{P}} \in \mathrm{BM}_{d}$ is allowed to be equal to $\boldsymbol{P}$ and thus $\boldsymbol{P} \in \mathrm{BM}_{d}$.

Let $\widetilde{\boldsymbol{P}}^{m}:=\left(\widetilde{p}^{m}(k, i ; \ell, j)\right)_{(k, i),(l, j) \in \mathbb{F}}, m \in \mathbb{N}$ denote the $m$ th power of $\widetilde{\boldsymbol{P}}$. For $k, \ell \in \mathbb{Z}_{+}$, let $\widetilde{\boldsymbol{P}}^{m}(k ; \ell):=\left(\widetilde{p}^{m}(k, i ; \ell, j)\right)_{i, j \in \mathbb{D}}$ denote the $(k, \ell)$ th block of $\widetilde{\boldsymbol{P}}^{m}$. We then have the following result.

Theorem 4.1 Suppose that (i) $\boldsymbol{P} \prec_{d} \widetilde{\boldsymbol{P}} \in \mathrm{BM}_{d}$ and $\widetilde{\boldsymbol{P}}$ is irreducible; and that (ii) there exist a constant $b \in(0, \infty)$, a column vector $\boldsymbol{v}=(v(k, i))_{(k, i) \in \mathbb{F}} \in \mathrm{BI}_{d}$ with $\boldsymbol{v} \geq \boldsymbol{e}$ and a nondecreasing differentiable concave function $\phi:[1, \infty) \rightarrow(0, \infty)$ with $\lim _{t \rightarrow \infty} \phi^{\prime}(t)=0$ such that, for some $M \in \mathbb{N}$ and $K \in \mathbb{Z}_{+}$,

$$
\begin{aligned}
\widetilde{\boldsymbol{P}}^{M} \boldsymbol{v} & \leq \boldsymbol{v}-\phi \circ \boldsymbol{v}+b \mathbf{1}_{K}, \\
\widetilde{\boldsymbol{P}}^{M}(K ; 0) \boldsymbol{e} & >\mathbf{0} .
\end{aligned}
$$


Let $B$ denote a positive constant such that

$$
B \cdot \widetilde{\boldsymbol{P}}^{M}(K ; 0) \boldsymbol{e} \geq b \boldsymbol{e} .
$$

Under these conditions, the following bound holds for all $m, n \in \mathbb{N}$.

$$
\left\|_{(n)} \boldsymbol{\pi}_{n}-\boldsymbol{\pi}\right\| \leq \frac{8\left\{c_{\phi, B}(1)\right\}^{-1}}{r_{\phi} \circ c_{\phi, B}(m-1)}\{v(1, \varpi)+B\}+2 m M b \sum_{i \in \mathbb{D}} \frac{1}{\phi \circ v(n, i)},
$$

where

$$
c_{\phi, B}(x)=\frac{\phi(1)}{\phi(B+1)} x, \quad x \geq 0 .
$$

In addition, if $K=0$, then, for all $m, n \in \mathbb{N}$,

$$
\left\|{ }_{(n)} \boldsymbol{\pi}_{n}-\boldsymbol{\pi}\right\| \leq \frac{8}{r_{\phi}(m-1)} v(1, \varpi)+2 m M b \sum_{i \in \mathbb{D}} \frac{1}{\phi \circ v(n, i)},
$$

which holds without (4.2).

Remark 4.1 Although the condition (4.2) ensures the existence of a constant $B \in(0, \infty)$ satisfying (4.3), it may seem that the condition (4.2) weakens the applicability of Theorem 4.1. However, that is not necessarily the case. To verify this, we assume that all the conditions of Theorem 4.1 are satisfied, except for (4.2), and that $\widetilde{\boldsymbol{P}}$ is aperiodic. Note here that the aperiodicity of $\widetilde{\boldsymbol{P}}$ does not make any restriction because $(\boldsymbol{I}+\boldsymbol{P}) / 2$ and $(\boldsymbol{I}+\widetilde{\boldsymbol{P}}) / 2$ are aperiodic and

$$
\begin{aligned}
\boldsymbol{\pi}(\boldsymbol{I}+\boldsymbol{P}) / 2 & =\boldsymbol{\pi}, & \tilde{\boldsymbol{\pi}}(\boldsymbol{I}+\widetilde{\boldsymbol{P}}) / 2 & =\widetilde{\boldsymbol{\pi}} \\
(\boldsymbol{I}+\boldsymbol{P}) / 2 & \prec_{d}(\boldsymbol{I}+\widetilde{\boldsymbol{P}}) / 2, & (\boldsymbol{I}+\widetilde{\boldsymbol{P}}) / 2 & \in \mathrm{BM}_{d} .
\end{aligned}
$$

We also assume the following mild condition.

$$
\lim _{k \rightarrow \infty} \phi \circ v(k, i)=\infty \quad \text { for all } i \in \mathbb{D} .
$$

Under these conditions, let $\left\{b_{m} ; m \in \mathbb{N}\right\}$ and $\left\{K_{m} ; m \in \mathbb{N}\right\}$ denote sequences such that $b_{1}=b$, $K_{1}=K$ and, for $m=2,3, \ldots$,

$$
\begin{aligned}
b_{m} & =\left\{b+b_{m-1}-(1-\lambda) \min _{i \in \mathbb{D}} \phi \circ v(0, i)\right\} \vee 0, \\
K_{m} & =\inf \left\{k \in \mathbb{N} ;\left(b+b_{m-1}\right) \boldsymbol{e}-(1-\lambda) \phi \circ \boldsymbol{v}(k) \leq \mathbf{0}\right\}-1,
\end{aligned}
$$

where $\lambda \in(0,1)$ is fixed arbitrarily. It then follows from (4.1) that

$$
\widetilde{\boldsymbol{P}}^{m M} \boldsymbol{v} \leq \boldsymbol{v}-\lambda \phi \circ \boldsymbol{v}+b_{m} \mathbf{1}_{K_{m}}, \quad m \in \mathbb{N} .
$$

In addition, the aperiodicity of $\widetilde{\boldsymbol{P}}$ implies that for any $m \in \mathbb{N}$ there exists some $\nu_{0}\left(K_{m}\right) \in \mathbb{N}$ such that $\widetilde{\boldsymbol{P}}^{\nu}\left(K_{m} ; 0\right) \boldsymbol{e}>\mathbf{0}$ for all $\nu \geq \nu_{0}\left(K_{m}\right)$ (see, e.g., [2, Chapter 2, Theorem 4.3]). As a result, if there exists some $m \in \mathbb{N}$ such that $\nu_{0}\left(K_{m}\right) \leq m M$, then we can obtain a bound similar to (4.4) by using Theorem 4.1 . 
Proof of Theorem 4.1. Let $\left\{\left(\widetilde{X}_{\nu}, \widetilde{J}_{\nu}\right) ; \nu \in \mathbb{Z}_{+}\right\}$denote a block-monotone Markov chain with state space $\mathbb{F}$ and transition probability matrix $\widetilde{\boldsymbol{P}}$. Conditions (i) and (ii) imply that $\widetilde{\boldsymbol{P}}$ is irreducible and positive recurrent and thus has the unique stationary probability vector, denoted by $\tilde{\boldsymbol{\pi}}=(\tilde{\pi}(k, i))_{(k, i) \in \mathbb{F}}$. It follows from $\boldsymbol{P} \prec_{d} \widetilde{\boldsymbol{P}} \in \mathrm{BM}_{d}$ and [18, Proposition 2.3] that $\boldsymbol{\pi} \prec_{d} \tilde{\boldsymbol{\pi}}$ and thus

$$
\sum_{k=0}^{\infty} \widetilde{\pi}(k, i)=\sum_{k=0}^{\infty} \pi(k, i)=\varpi(i), \quad i \in \mathbb{D} .
$$

Following the last part of the proof of [18, Theorem 4.1], we have

$$
\left\|_{(n)} \boldsymbol{p}_{n}^{m}(0, \varpi)-\boldsymbol{p}^{m}(0, \varpi)\right\| \leq 2 m \sum_{i \in \mathbb{D}}(n) \widetilde{\pi}_{n}(n, i), \quad m \in \mathbb{N} .
$$

It follows from (4.8) and the triangle inequality that, for $m \in \mathbb{N}$,

$$
\begin{aligned}
\left\|_{(n)} \boldsymbol{\pi}_{n}-\boldsymbol{\pi}\right\| & \leq\left\|\boldsymbol{p}^{m M}(0, \varpi)-\boldsymbol{\pi}\right\|+\left\|_{(n)} \boldsymbol{p}_{n}^{m M}(0, \varpi)-{ }_{(n)} \boldsymbol{\pi}_{n}\right\| \\
& \quad+\left\|_{(n)} \boldsymbol{p}_{n}^{m M}(0, \varpi)-\boldsymbol{p}^{m M}(0, \varpi)\right\| \\
& \leq\left\|\boldsymbol{p}^{m M}(0, \varpi)-\boldsymbol{\pi}\right\|+\left\|_{(n)} \boldsymbol{p}_{n}^{m M}(0, \varpi)-{ }_{(n)} \boldsymbol{\pi}_{n}\right\|+2 m M \sum_{i \in \mathbb{D}}{ }_{(n)} \widetilde{\pi}_{n}(n, i() 4
\end{aligned}
$$

For $n \in \mathbb{N}$, let $\left.{ }_{(n)} \widetilde{\boldsymbol{P}}_{n}:={ }_{\left({ }_{(n)}\right.} \widetilde{p}_{n}(k, i ; \ell, j)\right)_{(k, i),(\ell, j) \in \mathbb{F}}$ denote a stochastic matrix such that, for $i, j \in \mathbb{D}$,

$$
{ }_{(n)} \widetilde{p}_{n}(k, i ; \ell, j)= \begin{cases}\widetilde{p}(k, i ; \ell, j), & k \in \mathbb{Z}_{+}, \ell=0,1, \ldots, n-1, \\ \sum_{m=n}^{\infty} \widetilde{p}(k, i ; m, j), & k \in \mathbb{Z}_{+}, \ell=n, \\ 0, & \text { otherwise. }\end{cases}
$$

Note that ${ }_{(n)} \widetilde{\boldsymbol{P}}_{n}$ is the LC-block-augmented truncation of $\widetilde{\boldsymbol{P}}$. Note also that ${ }_{(n)} \widetilde{\boldsymbol{P}}_{n} \prec_{d} \widetilde{\boldsymbol{P}} \in \mathrm{BM}_{d}$. Thus, it follows from [18, Proposition 2.3] that $\left.{ }_{\left({ }_{(n)}\right.} \widetilde{\boldsymbol{P}}_{n}\right)^{M} \prec_{d} \widetilde{\boldsymbol{P}}^{M}$. Using this relation together with $v \in \mathrm{BI}_{d}$ and [18, Remark 2.1], we obtain

$$
\left({ }_{(n)} \widetilde{\boldsymbol{P}}_{n}\right)^{M} \boldsymbol{v} \leq \widetilde{\boldsymbol{P}}^{M} \boldsymbol{v}
$$

Combining (4.1) and (4.10), we have

$$
\left.{ }_{\left({ }_{(n)}\right.} \widetilde{\boldsymbol{P}}_{n}\right)^{M} \boldsymbol{v} \leq \widetilde{\boldsymbol{P}}^{M} \boldsymbol{v} \leq \boldsymbol{v}-\phi \circ \boldsymbol{v}+b \mathbf{1}_{K} .
$$

Pre-multiplying this inequality by ${ }_{(n)} \widetilde{\boldsymbol{\pi}}_{n}$ yields

$$
{ }_{(n)} \widetilde{\pi}_{n}(n, i) \leq \frac{b}{\phi \circ v(n, i)}, \quad i \in \mathbb{D} .
$$

Substituting (4.11) into (4.9) results in

$$
\begin{gathered}
\left\|{ }_{(n)} \boldsymbol{\pi}_{n}-\boldsymbol{\pi}\right\| \leq\left\|\boldsymbol{p}^{m M}(0, \varpi)-\boldsymbol{\pi}\right\|+\left\|_{(n)} \boldsymbol{p}_{n}^{m M}(0, \varpi)-{ }_{(n)} \boldsymbol{\pi}_{n}\right\| \\
+2 m M b \sum_{i \in \mathbb{D}} \frac{1}{\phi \circ v(n, i)} .
\end{gathered}
$$


We now define $\left\{\left(\widetilde{X}_{\nu}^{(M)}, \widetilde{J}_{\nu}^{(M)}\right) ; \nu \in \mathbb{Z}_{+}\right\}$as the $M$-skeleton of the Markov chain $\left\{\left(\widetilde{X}_{\nu}, \widetilde{J}_{\nu}\right) ; \nu \in\right.$ $\left.\mathbb{Z}_{+}\right\}$with transition probability matrix $\widetilde{\boldsymbol{P}}$, i.e.,

$$
\widetilde{X}_{\nu}^{(M)}=\widetilde{X}_{\nu M}, \quad \widetilde{J}_{\nu}^{(M)}=\widetilde{J}_{\nu M}, \quad \nu \in \mathbb{Z}_{+} .
$$

Clearly, the $M$-skeleton $\left\{\left(\widetilde{X}_{\nu}^{(M)}, \widetilde{J}_{\nu}^{(M)}\right)\right\}$ evolves according to $\widetilde{\boldsymbol{P}}^{M}$. We also define $\widetilde{\tau}_{0}^{(M)+}=$ $\inf \left\{\nu \in \mathbb{N} ; \widetilde{X}_{\nu}^{(M)}=\widetilde{X}_{\nu M}=0\right\}$. Proceeding as in the derivation of the first inequality for $\left\|\boldsymbol{p}^{m}(k, \varpi)-\boldsymbol{\pi}\right\|_{\boldsymbol{v}}$ at page 97 of [18] (replacing $\boldsymbol{v}$ by $\boldsymbol{e}$ as in the derivation of (3.17)) and using (4.7), we obtain

$$
\begin{aligned}
\left\|\boldsymbol{p}^{m M}(k, \varpi)-\boldsymbol{\pi}\right\| \leq & 2 \sum_{i \in \mathbb{D}} \varpi(i) \widetilde{\mathrm{P}}_{(k, i)}\left(\widetilde{\tau}_{0}^{(M)+}>m\right) \\
& +2 \sum_{(\ell, j) \in \mathbb{F}} \widetilde{\pi}(\ell, j) \widetilde{\mathrm{P}}_{(\ell, j)}\left(\widetilde{\tau}_{0}^{(M)+}>m\right), \quad k \in \mathbb{Z}_{+}, m \in \mathbb{N},
\end{aligned}
$$

where $\widetilde{\mathrm{P}}_{(k, i)}(\cdot)=\mathrm{P}\left(\cdot \mid \widetilde{X}_{0}=k, \widetilde{J}_{0}=i\right)$ for $(k, i) \in \mathbb{F}$. In addition, by Markov's inequality,

$$
\widetilde{\mathrm{P}}_{(\ell, j)}\left(\widetilde{\tau}_{0}^{(M)+}>m\right) \leq \frac{1}{r_{\phi}(m-1)} \widetilde{\mathrm{E}}_{(\ell, j)}\left[r_{\phi}\left(\widetilde{\tau}_{0}^{(M)+}-1\right)\right], \quad m \in \mathbb{N},(\ell, j) \in \mathbb{F},
$$

where

$$
\widetilde{\mathrm{E}}_{(\ell, j)}[\cdot]=\widetilde{\mathrm{E}}\left[\cdot \mid \widetilde{X}_{0}=\ell, \widetilde{J}_{0}=j\right], \quad(\ell, j) \in \mathbb{F} .
$$

Substituting (4.14) into (4.13), we have, for $m \in \mathbb{N}$,

$$
\begin{aligned}
& \left\|\boldsymbol{p}^{m M}(k, \varpi)-\boldsymbol{\pi}\right\| \\
& \quad \leq \frac{2}{r_{\phi}(m-1)}\left(\sum_{i \in \mathbb{D}} \varpi(i) \widetilde{\mathrm{E}}_{(k, i)}\left[r_{\phi}\left(\widetilde{\tau}_{0}^{(M)+}-1\right)\right]+\widetilde{\mathrm{E}}_{\widetilde{\boldsymbol{\pi}}}\left[r_{\phi}\left(\widetilde{\tau}_{0}^{(M)+}-1\right)\right]\right),
\end{aligned}
$$

where

$$
\widetilde{\mathrm{E}}_{\widetilde{\pi}}[\cdot]=\sum_{(k, i) \in \mathbb{F}} \widetilde{\pi}(k, i) \widetilde{\mathrm{E}}_{(k, i)}[\cdot] .
$$

It should be noted that if $K=0$ then Lemma 3.1 yields

$$
\begin{aligned}
\widetilde{\mathrm{E}}_{(k, i)}\left[r_{\phi}\left(\widetilde{\tau}_{0}^{(M)+}-1\right)\right] & \leq v(k \vee 1, i), \quad(k, i) \in \mathbb{F}, \\
\widetilde{\mathrm{E}}_{\widetilde{\pi}}\left[r_{\phi}\left(\widetilde{\tau}_{0}^{(M)+}-1\right)\right] \leq v(k \vee 1, \varpi), & k \in \mathbb{Z}_{+} .
\end{aligned}
$$

Combining (4.15) with (4.16) and (4.17), we obtain

$$
\left\|\boldsymbol{p}^{m M}(k, \varpi)-\boldsymbol{\pi}\right\| \leq \frac{4}{r_{\phi}(m-1)} v(k \vee 1, \varpi), \quad m \in \mathbb{N} .
$$

Similarly, we have

$$
\left\|{ }_{(n)} \boldsymbol{p}_{n}^{m M}(k, \varpi)-{ }_{(n)} \boldsymbol{\pi}_{n}\right\| \leq \frac{4}{r_{\phi}(m-1)} v(k \vee 1, \varpi), \quad m, n \in \mathbb{N} .
$$


Substituting (4.18) and (4.19) into (4.12) yields the bound (4.6) in the special case where $K=0$. In what follows, we consider the general case, where $K \in \mathbb{N}$. In fact, the following inequality holds (which is proved later):

$$
\widetilde{\boldsymbol{P}}^{M} \widetilde{\boldsymbol{v}} \leq \widetilde{\boldsymbol{v}}-\widetilde{\phi} \circ \widetilde{\boldsymbol{v}}+\widetilde{b} \mathbf{1}_{0}
$$

where

$$
\begin{aligned}
\widetilde{\phi}(x) & =\frac{\phi(1)}{\phi(B+1)} \phi(x), \quad x \geq 1, \\
\widetilde{b} & =b+B, \\
\widetilde{v}(k, i) & = \begin{cases}v(0, i), & k=0, i \in \mathbb{D}, \\
v(k, i)+B, & k \in \mathbb{N}, i \in \mathbb{D} .\end{cases}
\end{aligned}
$$

Since $\widetilde{\boldsymbol{v}} \in \mathrm{BI}_{d}$ and $\left.{ }_{\left({ }_{n}\right)} \widetilde{\boldsymbol{P}}_{n}\right)^{M} \prec_{d} \widetilde{\boldsymbol{P}}^{M}$, we have $\left({ }_{(n)} \widetilde{\boldsymbol{P}}_{n}\right)^{M} \widetilde{\boldsymbol{v}} \leq \widetilde{\boldsymbol{P}}^{M} \widetilde{\boldsymbol{v}}$ (see [18, Remark 2.1]). It follows from this inequality and (4.20) that

$$
\left({ }_{(n)} \widetilde{\boldsymbol{P}}_{n}\right)^{M} \widetilde{\boldsymbol{v}} \leq \widetilde{\boldsymbol{P}}^{M} \widetilde{\boldsymbol{v}} \leq \widetilde{\boldsymbol{v}}-\widetilde{\phi} \circ \widetilde{\boldsymbol{v}}+\widetilde{b} \mathbf{1}_{0} .
$$

Note here that $\widetilde{\phi}:[1, \infty) \rightarrow(0, \infty)$ is a nondecreasing differentiable concave function such that $\lim _{t \rightarrow \infty} \widetilde{\phi}^{\prime}(t)=0$. The inequality (4.24) implies that the case where $K \in \mathbb{N}$ is reduced to the special case where $K=0$.

To follow the proof of the case where $K=0$, we define two functions $H_{\tilde{\phi}}$ and $r_{\widetilde{\phi}}$, which correspond to the functions $H_{\phi}$ and $r_{\phi}$, respectively. Let $H_{\widetilde{\phi}}$ denote a function on $[1, \infty)$ such that

$$
H_{\widetilde{\phi}}(x)=\int_{1}^{x} \frac{\mathrm{d} y}{\widetilde{\phi}(y)}=\left\{c_{\phi, B}(1)\right\}^{-1} H_{\phi}(x), \quad x \geq 1,
$$

where the second equality results from (3.4), (4.5) and (4.21). Furthermore, let $r_{\tilde{\phi}}$ denote a function on $[0, \infty)$ such that

$$
r_{\tilde{\phi}}(x)=\widetilde{\phi} \circ H_{\tilde{\phi}}^{-1}(x), \quad x \geq 0 .
$$

We then have

$$
\begin{aligned}
r_{\widetilde{\phi}}(x) & =c_{\phi, B}(1) \cdot \phi \circ H_{\widetilde{\phi}}^{-1}(x) \\
& =c_{\phi, B}(1) \cdot \phi \circ H_{\phi}^{-1}\left(c_{\phi, B}(x)\right) \\
& =c_{\phi, B}(1) \cdot r_{\phi} \circ c_{\phi, B}(x), \quad x \geq 0,
\end{aligned}
$$

where the first, second and third equalities follows from (4.21), (4.25) and (3.5), respectively. In addition, following the derivation of (4.18) and using $\widetilde{\boldsymbol{v}}$ in (4.23) and $r_{\tilde{\phi}}$ in (4.26) (instead of $\boldsymbol{v}$ and $r_{\phi}$ ), we obtain

$$
\begin{aligned}
\left\|\boldsymbol{p}^{m M}(k, \varpi)-\boldsymbol{\pi}\right\| & \leq \frac{4}{r_{\widetilde{\phi}}(m-1)} \widetilde{v}(k \vee 1, \varpi) \\
& =\frac{4\left\{c_{\phi, B}(1)\right\}^{-1}}{r_{\phi} \circ c_{\phi, B}(m-1)}\{v(k \vee 1, \varpi)+B\}, \quad k \in \mathbb{Z}_{+} .
\end{aligned}
$$


Similarly,

$$
\left\|_{(n)} \boldsymbol{p}_{n}^{m M}(k, \varpi)-{ }_{(n)} \boldsymbol{\pi}_{n}\right\| \leq \frac{4\left\{c_{\phi, B}(1)\right\}^{-1}}{r_{\phi} \circ c_{\phi, B}(m-1)}\{v(k \vee 1, \varpi)+B\}, \quad k \in \mathbb{Z}_{+}(4
$$

Substituting (4.27) and (4.28) into (4.12) yields (4.4).

It remains to prove that (4.20) holds. For $k \in \mathbb{Z}_{+}$, let $\boldsymbol{v}(k)=(v(k, i))_{i \in \mathbb{D}}$ and $\widetilde{\boldsymbol{v}}(k)=$ $(\widetilde{v}(k, i))_{i \in \mathbb{D}}$, i.e.,

$$
\boldsymbol{v}^{\top}=\left(\boldsymbol{v}^{\top}(0), \boldsymbol{v}^{\top}(1), \boldsymbol{v}^{\top}(2), \ldots\right), \quad \widetilde{\boldsymbol{v}}^{\top}=\left(\widetilde{\boldsymbol{v}}^{\top}(0), \widetilde{\boldsymbol{v}}^{\top}(1), \widetilde{\boldsymbol{v}}^{\top}(2), \ldots\right),
$$

where the superscript "T" denotes the transpose operator for vectors and matrices. It then follows from (4.1), (4.22) and (4.23) that

$$
\begin{aligned}
\sum_{\ell=0}^{\infty} \widetilde{\boldsymbol{P}}^{M}(0 ; \ell) \widetilde{\boldsymbol{v}}(\ell) & \leq \sum_{\ell=0}^{\infty} \widetilde{\boldsymbol{P}}^{M}(0 ; \ell) \boldsymbol{v}(\ell)+B \boldsymbol{e} \leq \boldsymbol{v}(0)-\phi \circ \boldsymbol{v}(0)+(b+B) \boldsymbol{e} \\
& =\widetilde{\boldsymbol{v}}(0)-\phi \circ \widetilde{\boldsymbol{v}}(0)+\widetilde{b} \boldsymbol{e} \leq \widetilde{\boldsymbol{v}}(0)-\widetilde{\phi} \circ \widetilde{\boldsymbol{v}}(0)+\widetilde{b} \boldsymbol{e}
\end{aligned}
$$

where the last inequality holds because $\phi(x) \geq \widetilde{\phi}(x)$ for $x \geq 1$ (see (4.21)).

It should be noted that $\widetilde{\boldsymbol{P}}^{M} \in \mathrm{BM}_{d}$ due to $\widetilde{\boldsymbol{P}} \in \mathrm{BM}_{d}$. Thus, $\widetilde{\boldsymbol{P}}^{M}(k ; 0) \geq \widetilde{\boldsymbol{P}}^{M}(K ; 0)$ for $k=0,1, \ldots, K$. Using this and (4.23), we have, for $k=1,2, \ldots, K$,

$$
\begin{aligned}
\sum_{\ell=0}^{\infty} \widetilde{\boldsymbol{P}}^{M}(k ; \ell) \widetilde{\boldsymbol{v}}(\ell) & =\sum_{\ell=0}^{\infty} \widetilde{\boldsymbol{P}}^{M}(k ; \ell) \boldsymbol{v}(\ell)+B\left\{\boldsymbol{e}-\widetilde{\boldsymbol{P}}^{M}(k ; 0) \boldsymbol{e}\right\} \\
& \leq \sum_{\ell=0}^{\infty} \widetilde{\boldsymbol{P}}^{M}(k ; \ell) \boldsymbol{v}(\ell)+B\left\{\boldsymbol{e}-\widetilde{\boldsymbol{P}}^{M}(K ; 0) \boldsymbol{e}\right\}
\end{aligned}
$$

Applying (4.1) and (4.23) to the right hand side of (4.30) yields, for $k=1,2, \ldots, K$,

$$
\begin{aligned}
\sum_{\ell=0}^{\infty} \widetilde{\boldsymbol{P}}^{M}(k ; \ell) \widetilde{\boldsymbol{v}}(\ell) & \leq \boldsymbol{v}(k)-\phi \circ \boldsymbol{v}(k)+b \boldsymbol{e}+B\left\{\boldsymbol{e}-\widetilde{\boldsymbol{P}}^{M}(K ; 0) \boldsymbol{e}\right\} \\
& =\widetilde{\boldsymbol{v}}(k)-\phi \circ \boldsymbol{v}(k)+b \boldsymbol{e}-B \widetilde{\boldsymbol{P}}^{M}(K ; 0) \boldsymbol{e}
\end{aligned}
$$

Note here that the nonnegative concave function $\phi:[1, \infty) \rightarrow(0, \infty)$ is log-concave and thus

$$
\begin{aligned}
\frac{\mathrm{d}}{\mathrm{d} x}\left(\frac{\phi(x-B)}{\phi(x)}\right) & =\frac{\phi(x-B)}{\phi(x)}\left(\frac{\phi^{\prime}(x-B)}{\phi(x-B)}-\frac{\phi^{\prime}(x)}{\phi(x)}\right) \\
& =\frac{\phi(x-B)}{\phi(x)}\left\{\frac{\mathrm{d}}{\mathrm{d} x} \log \phi(x-B)-\frac{\mathrm{d}}{\mathrm{d} x} \log \phi(x)\right\} \geq 0 .
\end{aligned}
$$

Therefore, it follows from (4.21) that

$$
\frac{\phi(x-B)}{\phi(x)} \geq \frac{\phi(1)}{\phi(B+1)}=\frac{\widetilde{\phi}(x)}{\phi(x)}, \quad x \geq B+1,
$$


which leads to

$$
\widetilde{\phi}(x) \leq \phi(x-B), \quad x \geq B+1 .
$$

In addition, from (4.23) and $v \geq e$, we have

$$
\widetilde{\boldsymbol{v}}(k)=\boldsymbol{v}(k)+B \boldsymbol{e} \geq(B+1) \boldsymbol{e}, \quad k \in \mathbb{N} .
$$

The inequalities (4.32) and (4.33) imply that

$$
\widetilde{\phi} \circ \widetilde{\boldsymbol{v}}(k) \leq \phi \circ \boldsymbol{v}(k), \quad k \in \mathbb{N},
$$

where $\widetilde{\phi} \circ \widetilde{\boldsymbol{v}}(k)=(\widetilde{\phi} \circ \widetilde{v}(k, i))_{i \in \mathbb{D}}$. Substituting (4.34) into (4.31) and using (4.3), we obtain

$$
\begin{aligned}
\sum_{\ell=0}^{\infty} \widetilde{\boldsymbol{P}}^{M}(k ; \ell) \widetilde{\boldsymbol{v}}(\ell) & \leq \widetilde{\boldsymbol{v}}(k)-\widetilde{\phi} \circ \widetilde{\boldsymbol{v}}(k)+\left\{b \boldsymbol{e}-B \widetilde{\boldsymbol{P}}^{M}(K ; 0) \boldsymbol{e}\right\} \\
& \leq \widetilde{\boldsymbol{v}}(k)-\widetilde{\phi} \circ \widetilde{\boldsymbol{v}}(k), \quad k=1,2, \ldots, K .
\end{aligned}
$$

Similarly, it follows from (4.1), (4.23) and (4.34) that, for $k=K+1, K+2, \ldots$,

$$
\begin{aligned}
\sum_{\ell=0}^{\infty} \widetilde{\boldsymbol{P}}^{M}(k ; \ell) \widetilde{\boldsymbol{v}}(\ell) & \leq \sum_{\ell=0}^{\infty} \widetilde{\boldsymbol{P}}^{M}(k ; \ell) \boldsymbol{v}(\ell)+B \boldsymbol{e} \leq \boldsymbol{v}(k)-\phi \circ \boldsymbol{v}(k)+B \boldsymbol{e} \\
& =\widetilde{\boldsymbol{v}}(k)-\phi \circ \boldsymbol{v}(k) \leq \widetilde{\boldsymbol{v}}(k)-\widetilde{\phi} \circ \widetilde{\boldsymbol{v}}(k) .
\end{aligned}
$$

As a result, combining (4.29), (4.35) and (4.36) results in (4.20).

\section{Applications to GI/G/1-type Markov chains}

In this section, we discuss the application of our results to block-monotone GI/G/1-type Markov chains. We first present a procedure for establishing an error bound for the LC-block-augmented truncation of a general block-monotone GI/G/1-type Markov chain. We then consider such a simple special case that the parameters of the error bound are specified.

\subsection{General case}

In this subsection, we consider a general block-monotone GI/G/1-type Markov chain, which satisfies the following assumption.

\section{Assumption 5.1}

(i) $\boldsymbol{P} \in \mathrm{BM}_{d}$ is an irreducible GI/G/1-type transition probability matrix such that

$$
\boldsymbol{P}=\left(\begin{array}{ccccc}
\boldsymbol{B}(0) & \boldsymbol{B}(1) & \boldsymbol{B}(2) & \boldsymbol{B}(3) & \ldots \\
\underline{\boldsymbol{A}}(-1) & \boldsymbol{A}(0) & \boldsymbol{A}(1) & \boldsymbol{A}(2) & \ldots \\
\underline{\boldsymbol{A}}(-2) & \boldsymbol{A}(-1) & \boldsymbol{A}(0) & \boldsymbol{A}(1) & \ldots \\
\underline{\boldsymbol{A}}(-3) & \boldsymbol{A}(-2) & \boldsymbol{A}(-1) & \boldsymbol{A}(0) & \ldots \\
\vdots & \vdots & \vdots & \vdots & \ddots
\end{array}\right)
$$


where $\underline{\boldsymbol{A}}(-k)=\sum_{\ell=-\infty}^{-k} \boldsymbol{A}(\ell)$ for $k \in \mathbb{Z}_{+}$and the diagonal blocks $\boldsymbol{A}(0)$ and $\boldsymbol{B}(0)$ are $d \times d$ matrices; and

(ii) $\boldsymbol{A}:=\sum_{k=-\infty}^{\infty} \boldsymbol{A}(k)$ is an irreducible stochastic matrix.

Remark 5.1 The GI/G/1-type transition probability matrix $\boldsymbol{P}$ in (5.1) above is equal to $\boldsymbol{P}$ in (5.1) of [18], though the latter has the block matrices $\{\boldsymbol{B}(0), \boldsymbol{B}(-1), \boldsymbol{B}(-2), \ldots\}$ in the first block column. Indeed, for all $k \in \mathbb{N}, \boldsymbol{B}(-k)$ must be equal to $\underline{\boldsymbol{A}}(-k)$ because $\boldsymbol{A}=$ $\boldsymbol{B}(-k)+\sum_{\ell=-k+1}^{\infty} \boldsymbol{A}(\ell)$ due to $\boldsymbol{P} \in \mathrm{BM}_{d}$ (see [18, Proposition 1.1]).

It follows from (5.1), $P \in \mathrm{BM}_{d}$ and [18, Proposition 1.1] that, for all $k \in \mathbb{Z}_{+}$,

$$
\boldsymbol{\Psi}=\sum_{m=0}^{\infty} \boldsymbol{P}(k ; m)=\sum_{\ell=0}^{\infty} \boldsymbol{B}(\ell)=\sum_{\ell=-\infty}^{\infty} \boldsymbol{A}(\ell)=\boldsymbol{A} .
$$

Recall here that $\varpi$ is the stationary probability vector of $\Psi$ and thus $\boldsymbol{A}$. Using $\varpi$, we define $\sigma$ as

$$
\sigma=\varpi \sum_{k=-\infty}^{\infty} k \boldsymbol{A}(k) \boldsymbol{e}
$$

We then assume the following.

Assumption $5.2 \sigma<0$.

Under Assumptions 5.1 and 5.2, the GI/G/1-type transition probability matrix $\boldsymbol{P}$ in (5.1) is irreducible and positive recurrent (see, e.g., [1, Chapter XI, Proposition 3.1]) and thus has the unique stationary probability vector $\boldsymbol{\pi}=(\pi(k, i))_{(k, i) \in \mathbb{F}}$.

The subject of this subsection is to show how to obtain an upper bound for $\left\|_{(n)} \pi_{n}-\boldsymbol{\pi}\right\|$ by using Theorem 4.1. To apply Theorem 4.1 to $\boldsymbol{P}$ in (5.1), we have to prove that the drift condition (4.1) holds with $\widetilde{\boldsymbol{P}}$ being equal to $\boldsymbol{P}$, that is, $\boldsymbol{P}^{M} \boldsymbol{v} \leq \boldsymbol{v}-\phi \circ \boldsymbol{v}+b \mathbf{1}_{K}$. Unfortunately, since it is not easy to establish directly the drift condition on $\boldsymbol{P}^{M}$, we construct a modified transition probability matrix from $\boldsymbol{P}$ and establish a similar drift condition on the $M$ th power of the modified transition probability matrix. Using the similar drift condition, we derive the desired drift condition on $\boldsymbol{P}^{M}$.

We define the modified transition probability matrix mentioned above. For $N \in \mathbb{N}$, let $\boldsymbol{P}_{N}:=\left(p_{N}(k, i ; \ell, j)\right)_{(k, i),(\ell, j) \in \mathbb{F}}$ denote a stochastic matrix such that

$$
\boldsymbol{P}_{N}=\left(\begin{array}{ccccc}
\boldsymbol{B}(0) & \boldsymbol{B}(1) & \boldsymbol{B}(2) & \boldsymbol{B}(3) & \cdots \\
\boldsymbol{A}_{N}(-1) & \boldsymbol{A}_{N}(0) & \boldsymbol{A}_{N}(1) & \boldsymbol{A}_{N}(2) & \cdots \\
\vdots & \vdots & \vdots & \vdots & \ddots \\
\boldsymbol{A}_{N}(-N) & \boldsymbol{A}_{N}(-N+1) & \boldsymbol{A}_{N}(-N+2) & \boldsymbol{A}_{N}(-N+3) & \cdots \\
\boldsymbol{O} & \boldsymbol{A}_{N}(-N) & \boldsymbol{A}_{N}(-N+1) & \boldsymbol{A}_{N}(-N+2) & \cdots \\
\boldsymbol{O} & \boldsymbol{O} & \boldsymbol{A}_{N}(-N) & \boldsymbol{A}_{N}(-N+1) & \cdots \\
\vdots & \vdots & \vdots & \vdots & \ddots
\end{array}\right),
$$


where $\boldsymbol{A}_{N}(k), k \in \mathbb{Z}:=\{0, \pm 1, \pm 2, \ldots\}$ is given by

$$
\boldsymbol{A}_{N}(k)= \begin{cases}\boldsymbol{O}, & k \leq-N-1 \\ \underline{\boldsymbol{A}}(-N), & k=-N \\ \boldsymbol{A}(k), & k \geq-N+1\end{cases}
$$

It follows from (5.3) and Assumption 5.1 that $\boldsymbol{P} \prec{ }_{d} \boldsymbol{P}_{N} \in \mathrm{BM}_{d}$ for $N \in \mathbb{N}$. Furthermore, let

$$
\sigma_{N}=\varpi \sum_{k=-\infty}^{\infty} k \boldsymbol{A}_{N}(k) \boldsymbol{e}
$$

Assumption 5.2 implies that, for all sufficiently large $N \in \mathbb{N}$,

$$
\sigma_{N}<0
$$

In the rest of this subsection, we fix $N \in \mathbb{N}$ such that (5.6) holds. For $M \in \mathbb{N}$, we then define $\left\{\boldsymbol{A}_{N}^{* M}(k) ; k \in \mathbb{Z}\right\}$ as the $M$ th-fold convolution of $\left\{\boldsymbol{A}_{N}(k) ; k \in \mathbb{Z}\right\}$, i.e., $\boldsymbol{A}_{N}^{* 1}(k)=\boldsymbol{A}_{N}(k)$ for $k \in \mathbb{Z}$ and, for $M \geq 2$,

$$
\boldsymbol{A}_{N}^{* M}(k)=\sum_{\ell \in \mathbb{Z}} \boldsymbol{A}_{N}^{*(M-1)}(k-\ell) \boldsymbol{A}_{N}(\ell), \quad k \in \mathbb{Z} .
$$

From (5.4), we have $\boldsymbol{A}_{N}^{* M}(k)=\boldsymbol{O}$ for all $k \leq-L-1$, where $L=M N$. We also obtain the following result.

Lemma 5.1 There exists some $M_{0} \in \mathbb{N}$ such that

$$
\sum_{\ell=-L}^{\infty} \ell \boldsymbol{A}_{N}^{* M}(\ell) \boldsymbol{e}<\mathbf{0} \quad \text { for all } M \geq M_{0}
$$

Proof. We consider a Markov additive process $\left\{\left(Y_{\nu}, J_{\nu}\right) ; \nu \in \mathbb{Z}_{+}\right\}$with state space $\mathbb{Z} \times \mathbb{D}$ and kernel $\left\{\boldsymbol{A}_{N}(k) ; k \in \mathbb{Z}\right\}$, which evolves according to the following transition law.

$$
\mathrm{P}\left(Y_{\nu+1}-Y_{\nu}=k, J_{\nu+1}=j \mid J_{\nu}=i\right)=\left[\boldsymbol{A}_{N}(k)\right]_{i, j}, \quad k \in \mathbb{Z}, i, j \in \mathbb{D},
$$

where $[\cdot]_{i, j}$ denotes the $(i, j)$ th element of the matrix in the square brackets. Note here that $\varpi$ is the stationary probability vector of the background Markov chain $\left\{J_{\nu} ; \nu \in \mathbb{Z}_{+}\right\}$and thus $\sigma_{N}<0$ is the mean drift of the marginal process $\left\{Y_{\nu} ; \nu \in \mathbb{Z}_{+}\right\}$. In addition, it follows from $\sigma_{N}<0$ that $\mathrm{P}\left(\lim _{\nu \rightarrow \infty} Y_{\nu}=-\infty \mid Y_{0}=i\right)=1$ for all $i \in \mathbb{D}$ (see, e.g., [1, Chapter XI, Proposition 2.10]). Therefore, (5.7) holds for some $M_{0} \in \mathbb{N}$.

To proceed, we fix $M \geq M_{0}$ arbitrarily, where $M_{0}$ is some positive integer satisfying (5.7). We denote by $\boldsymbol{P}_{N}^{M}(k ; \ell), k, \ell \in \mathbb{Z}_{+}$the $(k, \ell)$ th block of the $M$ th power $\boldsymbol{P}_{N}^{M}$ of $\boldsymbol{P}_{N}$. It then follows from (5.3) that

$$
\boldsymbol{P}_{N}^{M}(k ; \ell)= \begin{cases}\boldsymbol{A}_{N}^{* M}(l-k), & k \geq L, \ell \geq k-L \\ \boldsymbol{O}, & k \geq L, \ell \leq k-L-1\end{cases}
$$


and thus

$$
\boldsymbol{P}_{N}^{M}=\left(\begin{array}{ccccc}
\boldsymbol{P}_{N}^{M}(0 ; 0) & \boldsymbol{P}_{N}^{M}(0 ; 1) & \boldsymbol{P}_{N}^{M}(0 ; 2) & \boldsymbol{P}_{N}^{M}(0 ; 3) & \cdots \\
\boldsymbol{P}_{N}^{M}(1 ; 0) & \boldsymbol{P}_{N}^{M}(1 ; 1) & \boldsymbol{P}_{N}^{M}(1 ; 2) & \boldsymbol{P}_{N}^{M}(1 ; 3) & \cdots \\
\vdots & \vdots & \vdots & \vdots & \ddots \\
\boldsymbol{P}_{N}^{M}(L-1 ; 0) & \boldsymbol{P}_{N}^{M}(L-1 ; 1) & \boldsymbol{P}_{N}^{M}(L-1 ; 2) & \boldsymbol{P}_{N}^{M}(L-1 ; 3) & \cdots \\
\boldsymbol{A}_{N}^{* M}(-L) & \boldsymbol{A}_{N}^{* M}(-L+1) & \boldsymbol{A}_{N}^{* M}(-L+2) & \boldsymbol{A}_{N}^{* M}(-L+3) & \cdots \\
\boldsymbol{O} & \boldsymbol{A}_{N}^{* M}(-L) & \boldsymbol{A}_{N}^{* M}(-L+1) & \boldsymbol{A}_{N}^{* M}(-L+2) & \cdots \\
\boldsymbol{O} & \boldsymbol{O} & \boldsymbol{A}_{N}^{* M}(-L) & \boldsymbol{A}_{N}^{* M}(-L+1) & \cdots \\
\vdots & \vdots & \vdots & \vdots & \ddots
\end{array}\right) .
$$

Since $\boldsymbol{P} \prec{ }_{d} \boldsymbol{P}_{N} \in \mathrm{BM}_{d}$, it follows from [18, Remark 2.1 and Proposition 2.3] that $\boldsymbol{P}^{M} \prec_{d} \boldsymbol{P}_{N}^{M}$, which implies that

$$
\boldsymbol{P}^{M} \boldsymbol{v} \leq \boldsymbol{P}_{N}^{M} \boldsymbol{v} \text { if } \boldsymbol{v} \in \mathrm{BI}_{d}
$$

Therefore, we show that $\boldsymbol{P}_{N}^{M}$ satisfies a drift condition similar to (4.1).

We now introduce a function $V:[0, \infty) \rightarrow[1, \infty)$, which plays a key role in establishing the drift condition on $\boldsymbol{P}_{N}^{M}$.

Assumption 5.3 There exists some $\alpha \in[0,1)$ such that, for any $\delta>0$,

$$
\lim _{k \rightarrow \infty} \frac{1}{V^{\prime}(k)} \sum_{\ell=\left\lfloor\delta k^{1-\alpha}\right\rfloor+1}^{\infty} V(k+\ell) \boldsymbol{A}_{N}^{* M}(\ell) \boldsymbol{e}=\mathbf{0}
$$

where $V:[0, \infty) \rightarrow[1, \infty)$ is an increasing, convex and log-concave function such that

(i) $\lim _{x \rightarrow \infty}(\log V(x)) / x=\log r_{A_{+}}$, where

$$
r_{A_{+}}=\sup \left\{z \geq 1 ; \sum_{\ell=0}^{\infty} z^{\ell} \boldsymbol{A}(l) \text { is finite }\right\}
$$

(ii) $V(x)$ is twice-differentiable for $x>0$;

(iii) $V^{\prime}(0):=\lim _{x \downarrow 0} V^{\prime}(x)>0$ and $\lim _{x \rightarrow \infty} V^{\prime}(x)=\infty$;

(iv) $V^{\prime \prime}(x) / V^{\prime}(x)$ is nonincreasing for $x>0$; and

(v) $\lim \sup _{\delta \downarrow 0} \lim _{x \rightarrow \infty} V^{\prime}\left(x+\delta x^{1-\alpha}\right) / V^{\prime}(x)=1$.

Remark 5.2 Appendix A provides a sufficient condition for Assumption 5.3 and the typical examples of $V$.

The key properties of the function $V$ are summarized in Lemmas 5.2 and 5.3 below. 
Lemma 5.2 Suppose that Assumption 5.3 is satisfied. It then holds that, for any $\alpha_{0} \in(\alpha, 1)$,

$$
V^{\prime}(x)=\exp \left\{o\left(x^{\alpha_{0}}\right)\right\}
$$

where $f(x)=o(g(x))$ represents $\lim _{x \rightarrow \infty} f(x) / g(x)=0$. It also holds that

$$
\log V(x)=o\left(x^{\alpha_{0}}\right)
$$

In addition,

$$
\lim _{x \rightarrow \infty} \frac{V^{\prime \prime}(x)}{V^{\prime}(x)}=0
$$

Proof. We first prove (5.11). Let $G$ denote a continuous function on $[0, \infty)$ such that

$$
G(x)=1-\frac{V^{\prime}(0)}{V^{\prime}(x)}, \quad x \geq 0 .
$$

Since $V^{\prime}$ is nondecreasing and $\lim _{x \rightarrow \infty} V^{\prime}(x)=\infty$ (due to the convexity of $V$ and condition (iii) of Assumption 5.3), $G$ is a distribution function. We now define $\bar{G}(x)=1-G(x)$ for $x \geq 0$, i.e.,

$$
\bar{G}(x)=\frac{V^{\prime}(0)}{V^{\prime}(x)}, \quad x \geq 0 .
$$

It then follows from condition (v) of Assumption 5.3 that

$$
\liminf _{\delta \downarrow} \lim _{x \rightarrow \infty} \frac{\bar{G}\left(x+\delta x^{1-\alpha}\right)}{\bar{G}(x)}=1,
$$

and thus, for any $\alpha_{0} \in(\alpha, 1)$,

$$
1 \geq \lim _{x \rightarrow \infty} \frac{\bar{G}\left(x+x^{1-\alpha_{0}}\right)}{\bar{G}(x)} \geq \liminf _{\delta \downarrow 0} \lim _{x \rightarrow \infty} \frac{\bar{G}\left(x+\delta x^{1-\alpha}\right)}{\bar{G}(x)}=1 .
$$

Therefore, we have

$$
\lim _{x \rightarrow \infty} \frac{\bar{G}\left(x+x^{1-\alpha_{0}}\right)}{\bar{G}(x)}=1,
$$

which implies that the distribution function $G$ is $\left(1 / \alpha_{0}\right)$ th-order long-tailed [17, Definition 1.1 and Lemma A.3]. Combining this fact with [17, Lemma A.1 (i)] yields

$$
\bar{G}(x)=\exp \left\{-o\left(x^{\alpha_{0}}\right)\right\}
$$

Substituting (5.15) into (5.14) results in (5.11). Furthermore, using (5.11), we can readily obtain (5.12).

Finally, we prove (5.13) by contradiction. To this end, we assume that (5.13) does not hold, i.e., there exist some $\delta>0$ and $x_{0}:=x_{0}(\delta)>0$ such that

$$
\frac{\mathrm{d}}{\mathrm{d} x} \log V^{\prime}(x)=\frac{V^{\prime \prime}(x)}{V^{\prime}(x)} \geq \delta \quad \text { for all } x>x_{0},
$$


and thus $\log V^{\prime}(x)-\log V^{\prime}\left(x_{0}\right) \geq \delta\left(x-x_{0}\right)$ for $x \geq x_{0}$, which yields

$$
\frac{V^{\prime}(x)}{V^{\prime}\left(x_{0}\right)} \geq \exp \left\{\delta\left(x-x_{0}\right)\right\}, \quad x \geq x_{0} .
$$

Therefore, we have

$$
V(x) \geq V\left(x_{0}\right)+\frac{V^{\prime}\left(x_{0}\right)}{\delta}\left(\mathrm{e}^{\delta\left(x-x_{0}\right)}-1\right), \quad x \geq x_{0} .
$$

From this inequality, we obtain $\lim \inf _{x \rightarrow \infty} \log V(x) / x \geq \delta$, which is inconsistent with (5.12).

Remark 5.3 Equation (5.12) shows that $\log V(x)=o(x)$. Thus, from condition (i) of Assumption 5.3, we have $r_{A_{+}}=1$, i.e., $\left\{\boldsymbol{A}(k) ; k \in \mathbb{Z}_{+}\right\}$is heavy-tailed.

Remark 5.4 As mentioned in Remark 5.1, Masuyama [18] considered the same block-monotone GI/G/1-type transition probability matrix as $\boldsymbol{P}$ in (5.1). However, Masuyama [18] assumed that $r_{A_{+}}>1$, i.e., $\left\{\boldsymbol{A}(k) ; k \in \mathbb{Z}_{+}\right\}$is light-tailed, which implies that $\left\{\boldsymbol{B}(k) ; k \in \mathbb{Z}_{+}\right\}$is also lighttailed. Indeed, since $\boldsymbol{P} \in \mathrm{BM}_{d}$, it holds that $\sum_{\ell=k}^{\infty} \boldsymbol{B}(\ell) \leq \sum_{\ell=k}^{\infty} \boldsymbol{A}(\ell)$ for all $k \in \mathbb{Z}_{+}$, which yields, for $z \in\left[1, r_{A_{+}}\right)$,

$$
\begin{aligned}
\sum_{k=0}^{\infty} z^{k} \boldsymbol{B}(k) & =\sum_{\ell=0}^{\infty} \boldsymbol{B}(\ell)+\sum_{\ell=1}^{\infty}\left(z^{\ell}-1\right) \boldsymbol{B}(\ell) \\
& =\sum_{\ell=0}^{\infty} \boldsymbol{B}(\ell)+\sum_{k=1}^{\infty}\left(z^{k}-z^{k-1}\right) \sum_{\ell=k}^{\infty} \boldsymbol{B}(\ell) \\
& \leq \sum_{\ell=0}^{\infty} \boldsymbol{A}(\ell)+\sum_{k=1}^{\infty}\left(z^{k}-z^{k-1}\right) \sum_{\ell=k}^{\infty} \boldsymbol{A}(\ell)=\sum_{k=0}^{\infty} z^{k} \boldsymbol{A}(k) .
\end{aligned}
$$

Thus, if $r_{A_{+}}>1$, then $r_{B_{+}}:=\sup \left\{z \geq 1 ; \sum_{\ell=0}^{\infty} z^{\ell} \boldsymbol{B}(l)\right.$ is finite $\} \geq r_{A_{+}}>1$. It is known (see [8, Theorem 2.2] and [14, Theorem 3.1]) that, under Assumptions 5.1] and 5.2] $\min \left(r_{A_{+}}, r_{B_{+}}\right)>$ 1 if and only if the GI/G/1-type transition probability matrix $\boldsymbol{P}$ in (5.1) satisfies the geometric drift condition (see Remark 3.1). On the other hand, Assumption 5.3 together with Assumptions 5.1 and 5.2 implies that the GI/G/1-type transition probability matrix $\boldsymbol{P}$ in (5.1) is positive recurrent but does not satisfy the geometric drift condition because $r_{A_{+}}=1$, i.e., $\left\{\boldsymbol{A}(k) ; k \in \mathbb{Z}_{+}\right\}$is heavy-tailed (see Remark 5.3). It is also known (see [11, Theorem 3.1]) that if $\left\{\boldsymbol{A}(k) ; k \in \mathbb{Z}_{+}\right\}$is heavy-tailed then so is the stationary probability vector of the ergodic GI/G/1-type transition probability matrix $\boldsymbol{P}$. Such GI/G/1-type transition probability matrices typically arise from BMAP/GI/1 queues with subexponential service times and/or batch sizes, and these Markov chains have the subexponential stationary probability vectors under some mild technical conditions [15, 19] (see also [9, 16]). 
Lemma 5.3 If Assumption 5.3 holds, then, for any $\varepsilon \in(0,1)$, there exist some $\delta_{0}:=\delta_{0}(\varepsilon)>0$ and positive integer $K_{0}:=K_{0}\left(\delta_{0}, L\right) \geq L$ such that

$$
\begin{aligned}
V^{\prime}\left(k+\delta_{0} k^{1-\alpha}\right) & \leq(1+\varepsilon) V^{\prime}(k), & & k \geq K_{0}+1, \\
V^{\prime}(k-L) & \geq(1-\varepsilon) V^{\prime}(k), & & k \geq K_{0}+1 .
\end{aligned}
$$

Proof. Recall that $V^{\prime}$ is nondecreasing. Thus, it follows from condition (v) of Assumption 5.3 that

$$
1 \geq \lim _{x \rightarrow \infty} \frac{V^{\prime}(x-L)}{V^{\prime}(x)}=\lim _{y \rightarrow \infty} \frac{V^{\prime}(y)}{V^{\prime}(y+L)} \geq \liminf _{\delta \downarrow 0} \lim _{y \rightarrow \infty} \frac{V^{\prime}(y)}{V^{\prime}\left(y+\delta y^{1-\alpha}\right)}=1,
$$

which leads to

$$
\lim _{x \rightarrow \infty} \frac{V^{\prime}(x-L)}{V^{\prime}(x)}=1 .
$$

In addition, condition (v) of Assumption 5.3 implies that, for any $\varepsilon \in(0,1)$,

$$
\lim _{x \rightarrow \infty} \frac{V^{\prime}\left(x+\delta_{0} x^{1-\alpha}\right)}{V^{\prime}(x)} \leq 1+2 \varepsilon
$$

where $\delta_{0}>0$ is sufficiently small depending on $\varepsilon \in(0,1)$. Consequently, (5.18) and (5.19) show that the statement of this lemma is true.

To establish the drift condition on $\boldsymbol{P}_{N}^{M}$, we estimate $\sum_{\ell=0}^{\infty} \boldsymbol{P}_{N}^{M}(k ; \ell) V(\ell) \boldsymbol{e}$ for sufficiently large $k$ 's by using Lemmas 5.1 and 5.3. Lemma 5.1 implies that there exist $\kappa>0$ and $\varepsilon \in(0,1)$ such that

$$
(1-\varepsilon) \sum_{\ell=-L}^{\infty} \ell \boldsymbol{A}_{N}^{* M}(\ell) \boldsymbol{e}+2 \varepsilon \sum_{\ell=0}^{\infty} \ell \boldsymbol{A}_{N}^{* M}(\ell) \boldsymbol{e} \leq-2 \kappa \boldsymbol{e} .
$$

Furthermore, according to (5.10), there exists some $K \in\left\{K_{0}, K_{0}+1, \ldots\right\}$ such that

$$
\frac{1}{V^{\prime}(k)} \sum_{\ell=\left\lfloor\delta_{0} k^{1-\alpha}\right\rfloor+1}^{\infty} V(k+\ell) \boldsymbol{A}_{N}^{* M}(\ell) \boldsymbol{e} \leq \kappa \boldsymbol{e} \quad \text { for all } k \geq K+1,
$$

where $\delta_{0}>0$ and $K_{0} \in\{L, L+1, \ldots\}$ are fixed such that (5.16) and (5.17) hold. From (5.20), (5.21) and Lemma 5.3, we obtain the following result.

Lemma 5.4 Suppose that Assumptions 5.1 5.2 and 5.3 are satisfied. Let $L=M N$, where $N \in \mathbb{N}$ and $M \in \mathbb{N}$ are fixed such that (5.6) and (5.7) hold. Furthermore, fix

(i) $\kappa>0$ and $\varepsilon \in(0,1)$ such that (5.20) holds;

(ii) $\delta_{0}>0$ and $K_{0} \in\{L, L+1, \ldots\}$ such that (5.16) and (5.17) hold; and

(iii) $K \in\left\{K_{0}, K_{0}+1, \ldots\right\}$ such that (5.21) holds. 
We then have

$$
\sum_{\ell=0}^{\infty} \boldsymbol{P}_{N}^{M}(k ; \ell) V(\ell) \boldsymbol{e}-V(k) \boldsymbol{e} \leq-\kappa V^{\prime}(k) \boldsymbol{e}, \quad k \geq K+1
$$

Proof. It follows from (5.8) and $K \geq K_{0} \geq L$ that, for $k \geq K+1$,

$$
\begin{aligned}
& \sum_{\ell=0}^{\infty} \boldsymbol{P}_{N}^{M}(k ; \ell) V(\ell) \boldsymbol{e}-V(k) \boldsymbol{e} \\
& \quad=\sum_{\ell=-L}^{\infty} \boldsymbol{A}_{N}^{* M}(\ell) V(k+\ell) \boldsymbol{e}-V(k) \boldsymbol{e} \\
& \quad=\sum_{\ell=\left\lfloor\delta_{0} k^{1-\alpha}\right\rfloor+1}^{\infty} \boldsymbol{A}_{N}^{* M}(\ell) V(k+\ell) \boldsymbol{e}+\sum_{\ell=-L}^{\left\lfloor\delta_{0} k^{1-\alpha}\right\rfloor} \boldsymbol{A}_{N}^{* M}(\ell) V(k+\ell) \boldsymbol{e}-V(k) \boldsymbol{e}
\end{aligned}
$$

where, by convention, any empty sum (which has no terms) is defined as zero. It also follows from the mean value theorem that, for any $k \in \mathbb{Z}_{+}$and $-L \leq \ell \leq \delta_{0} k^{1-\alpha}$, there exists some $\xi \in(0,1)$ such that

$$
V(k+\ell)=V(k)+\ell V^{\prime}(k+\xi \ell), \quad k \in \mathbb{Z}_{+},-L \leq \ell \leq \delta_{0} k^{1-\alpha} .
$$

Substituting (5.24) into the second term in the right hand side of (5.23) yields, for $k \geq K+1$,

$$
\begin{aligned}
\sum_{\ell=0}^{\infty} \boldsymbol{P}_{N}^{M}(k ; \ell) V(\ell) \boldsymbol{e}-V(k) \boldsymbol{e} \\
=\sum_{\ell=\left\lfloor\delta_{0} k^{1-\alpha}\right\rfloor+1}^{\infty} V(k+\ell) \boldsymbol{A}_{N}^{* M}(\ell) \boldsymbol{e}+\sum_{\ell=-L}^{\left\lfloor\delta_{0} k^{1-\alpha}\right\rfloor} V^{\prime}(k+\xi \ell) \ell \boldsymbol{A}_{N}^{* M}(\ell) \boldsymbol{e} \\
\quad+V(k) \sum_{\ell=-L}^{\left\lfloor\delta_{0} k^{1-\alpha}\right\rfloor} \boldsymbol{A}_{N}^{* M}(\ell) \boldsymbol{e}-V(k) \boldsymbol{e} \\
\leq \sum_{\ell=\left\lfloor\delta_{0} k^{1-\alpha}\right\rfloor+1}^{\infty} V(k+\ell) \boldsymbol{A}_{N}^{* M}(\ell) \boldsymbol{e}+\sum_{\ell=-L}^{\left\lfloor\delta_{0} k^{1-\alpha}\right\rfloor} V^{\prime}(k+\xi \ell) \ell \boldsymbol{A}_{N}^{* M}(\ell) \boldsymbol{e}
\end{aligned}
$$

where the inequality holds because $\sum_{\ell=-L}^{\left\lfloor\delta_{0} k^{1-\alpha}\right\rfloor} \boldsymbol{A}_{N}^{* M}(\ell) \boldsymbol{e} \leq \boldsymbol{e}$ for all $k \in \mathbb{Z}_{+}$. Furthermore, since $V^{\prime}$ is nondecreasing, we have

$$
\begin{aligned}
\sum_{\ell=-L}^{\left\lfloor\delta_{0} k^{1-\alpha}\right\rfloor} V^{\prime}(k+\xi \ell) \ell \boldsymbol{A}_{N}^{* M}(\ell) \boldsymbol{e} \leq & V^{\prime}(k-L) \sum_{\ell=-L}^{-1} \ell \boldsymbol{A}_{N}^{* M}(\ell) \boldsymbol{e} \\
& +V^{\prime}\left(k+\delta_{0} k^{1-\alpha}\right) \sum_{\ell=0}^{\left\lfloor\delta_{0} k^{1-\alpha}\right\rfloor} \ell \boldsymbol{A}_{N}^{* M}(\ell) \boldsymbol{e}, \quad k \geq K+1 .
\end{aligned}
$$


Applying (5.16) and (5.17) to the above inequality, we obtain, for $k \geq K+1$,

$$
\begin{aligned}
& \sum_{\ell=-L}^{\left\lfloor\delta_{0} k^{1-\alpha}\right\rfloor} V^{\prime}(k+\xi \ell) \ell \boldsymbol{A}_{N}^{* M}(\ell) \boldsymbol{e} \\
& \leq V^{\prime}(k)\left[(1-\varepsilon) \sum_{\ell=-L}^{-1} \ell \boldsymbol{A}_{N}^{* M}(\ell) \boldsymbol{e}+(1+\varepsilon) \sum_{\ell=0}^{\left\lfloor\delta_{0} k^{1-\alpha}\right\rfloor} \ell \boldsymbol{A}_{N}^{* M}(\ell) \boldsymbol{e}\right] \\
& =V^{\prime}(k)\left[(1-\varepsilon) \sum_{\ell=-L}^{\left\lfloor\delta_{0} k^{1-\alpha}\right\rfloor} \ell \boldsymbol{A}_{N}^{* M}(\ell) \boldsymbol{e}+2 \varepsilon \sum_{\ell=0}^{\left\lfloor\delta_{0} k^{1-\alpha}\right\rfloor} \ell \boldsymbol{A}_{N}^{* M}(\ell) \boldsymbol{e}\right] \\
& \leq V^{\prime}(k)\left[(1-\varepsilon) \sum_{\ell=-L}^{\infty} \ell \boldsymbol{A}_{N}^{* M}(\ell) \boldsymbol{e}+2 \varepsilon \sum_{\ell=0}^{\infty} \ell \boldsymbol{A}_{N}^{* M}(\ell) \boldsymbol{e}\right] \\
& \leq-2 \kappa V^{\prime}(k) \boldsymbol{e}, \quad[
\end{aligned}
$$

where we use (5.20) in the last inequality. Finally, substituting (5.21) and (5.26) into (5.25) yields (5.22).

We are now ready to establish the drift condition on $\boldsymbol{P}_{N}^{M}$ and thus $\boldsymbol{P}^{M}$. We fix

$$
\begin{aligned}
\boldsymbol{v}(k) & =V(k) \boldsymbol{e}, & & k \in \mathbb{Z}_{+}, \\
\phi(t) & =\kappa V^{\prime}\left(V^{-1}(t)\right), & & t \geq 1,
\end{aligned}
$$

where $V^{-1}$ denotes the inverse function of the increasing and differentiable function $V$ (see Assumption 5.3). Clearly, $v \in \mathrm{BM}_{d}$. Furthermore, it follows from (5.28) that

$$
\kappa V^{\prime}(k) \boldsymbol{e}=\phi \circ V(k) \boldsymbol{e}=\phi \circ \boldsymbol{v}(k), \quad k \in \mathbb{Z}_{+} .
$$

Substituting (5.27) and (5.29) into (5.22), we have

$$
\sum_{\ell=0}^{\infty} \boldsymbol{P}_{N}^{M}(k ; \ell) \boldsymbol{v}(\ell)-\boldsymbol{v}(k) \leq-\phi \circ \boldsymbol{v}(k), \quad k \geq K+1 .
$$

We also have

$$
\sum_{\ell=0}^{\infty} \boldsymbol{P}_{N}^{M}(k ; \ell) \boldsymbol{v}(\ell)-\boldsymbol{v}(k) \leq-\phi \circ \boldsymbol{v}(k)+b \boldsymbol{e}, \quad k=0,1, \ldots, K,
$$

where

$$
b=\inf \left\{x \geq 0 ; x \boldsymbol{e} \geq \sum_{\ell=0}^{\infty} \boldsymbol{P}_{N}^{M}(k ; \ell) \boldsymbol{v}(\ell)-\boldsymbol{v}(k)+\phi \circ \boldsymbol{v}(k), k=0,1, \ldots, K\right\} .
$$

Combining (5.30) and (5.31) yields

$$
\boldsymbol{P}_{N}^{M} \boldsymbol{v} \leq \boldsymbol{v}-\phi \circ \boldsymbol{v}+b \mathbf{1}_{K}
$$


From $v \in \mathrm{BM}_{d}$, (5.9) and (5.33), we obtain

$$
\boldsymbol{P}^{M} \boldsymbol{v} \leq \boldsymbol{v}-\phi \circ \boldsymbol{v}+b \mathbf{1}_{K}
$$

To use Theorem 4.1, it remains to show that the function $\phi$ in $(5.28)$ is qualified for the function $\phi$ appearing in Theorem 4.1, which is accomplished by Lemma 5.5 below.

Lemma 5.5 The function $\phi$ in (5.28) is nondecreasing, differentiable and concave. Furthermore, $\lim _{t \rightarrow \infty} \phi^{\prime}(t)=0$.

Proof of Lemma 5.5. Since $\left(V^{-1}\right)^{\prime}(t)=1 / V^{\prime}\left(V^{-1}(t)\right)$ for $t>1$, we obtain

$$
\phi^{\prime}(t)=\kappa V^{\prime \prime}\left(V^{-1}(t)\right) \cdot\left(V^{-1}\right)^{\prime}(t)=\kappa \frac{V^{\prime \prime}\left(V^{-1}(t)\right)}{V^{\prime}\left(V^{-1}(t)\right)}, \quad t>1 .
$$

It follows from (5.35), $V^{\prime}(x)>0$ and $V^{\prime \prime}(x) \geq 0$ for $x>0$ that

$$
\phi^{\prime}(t) \geq 0, \quad t>1
$$

which shows that $\phi$ is nondecreasing. It also follows from (5.35) and condition (iv) of Assumption 5.3 that $\phi^{\prime}$ is nonincreasing, which implies that $\phi$ is concave. In addition, since $\lim _{x \rightarrow \infty} V^{\prime}(x)=\infty$ (see condition (iii) of Assumption 5.3), we have $\lim _{x \rightarrow \infty} V(x)=\infty$ and thus $\lim _{t \rightarrow \infty} V^{-1}(t)=\infty$. Therefore, (5.35) together with (5.13) yields $\lim _{t \rightarrow \infty} \phi^{\prime}(t)=0$.

The following theorem is immediate from Theorem 4.1 with $\widetilde{\boldsymbol{P}}=\boldsymbol{P}$ and $\phi \circ \boldsymbol{v}(k)=$ $\kappa V^{\prime}(k) e(\operatorname{see}(5.29))$.

Theorem 5.1 Suppose that all the conditions of Lemma 5.4 are satisfied. Furthermore, suppose that $\boldsymbol{P}^{M}(K ; 0) \boldsymbol{e}>\mathbf{0}$, and fix $B \in(0, \infty)$ such that $B \boldsymbol{P}^{M}(K ; 0) \boldsymbol{e} \geq$ be, where $b$ is given in (5.32). Under these conditions, we have

$$
\left\|_{(n)} \boldsymbol{\pi}_{n}-\boldsymbol{\pi}\right\| \leq \frac{8\left\{c_{\phi, B}(1)\right\}^{-1}}{r_{\phi} \circ c_{\phi, B}(m-1)}(V(1)+B)+\frac{2 m M b d}{\kappa V^{\prime}(n)} \quad \text { for all } m, n \in \mathbb{N},
$$

where the composite function $r_{\phi} \circ c_{\phi, B}$ is given by (3.5), (4.5) and (5.28). In addition, if $K=0$, then

$$
\left\|_{(n)} \boldsymbol{\pi}_{n}-\boldsymbol{\pi}\right\| \leq \frac{8}{r_{\phi}(m-1)} V(1)+\frac{2 m M b d}{\kappa V^{\prime}(n)} \quad \text { for all } m, n \in \mathbb{N}
$$

Remark 5.5 Recall that $\lim _{x \rightarrow \infty} V^{\prime}(x)=\infty$ (see condition (iii) of Assumption 5.3) and $\lim _{t \rightarrow \infty} V^{-1}(t)=$ $\infty$ (see the proof of Lemma 5.5). Therefore, $\lim _{t \rightarrow \infty} \phi(t)=\lim _{t \rightarrow \infty} \kappa V^{\prime}\left(V^{-1}(t)\right)=\infty$, which leads to $\lim _{x \rightarrow \infty} r_{\phi}(x)=\infty$, as stated in Remark 3.2. Consequently, we can choose $m, n \in \mathbb{N}$ such that the error bounds (5.36) and (5.37) are reduced to any desired value less than two. 


\subsection{Special case}

In this subsection, we consider the LC-block-augmented truncation of a special block-monotone GI/G/1-type Markov chain, for which we establish an error bound with specified parameters in accordance with Theorem 5.1, To this end, in addition to Assumption 5.1, we assume that $\boldsymbol{P}$ in (5.1) is reduced to

$$
\boldsymbol{P}=\left(\begin{array}{ccccc}
\underline{\boldsymbol{A}}(0) & \boldsymbol{A}(1) & \boldsymbol{A}(2) & \boldsymbol{A}(3) & \ldots \\
\underline{\boldsymbol{A}}(-1) & \boldsymbol{A}(0) & \boldsymbol{A}(1) & \boldsymbol{A}(2) & \ldots \\
\underline{\boldsymbol{A}}(-2) & \boldsymbol{A}(-1) & \boldsymbol{A}(0) & \boldsymbol{A}(1) & \ldots \\
\underline{\boldsymbol{A}}(-3) & \boldsymbol{A}(-2) & \boldsymbol{A}(-1) & \boldsymbol{A}(0) & \ldots \\
\vdots & \vdots & \vdots & \vdots & \ddots
\end{array}\right)
$$

with

$$
\begin{aligned}
& \boldsymbol{A}(k)=\left(\begin{array}{cc}
0 & 2^{k-1} \\
2^{k-1} & 0
\end{array}\right), \quad k \leq-1, \\
& \boldsymbol{A}(k)=\frac{1}{2}\left(\begin{array}{cc}
0 & \frac{(k+1)^{-\beta_{1}}}{\zeta\left(\beta_{1}\right)} \\
\frac{(k+1)^{-\beta_{2}}}{\zeta\left(\beta_{2}\right)} & 0
\end{array}\right), \quad k \in \mathbb{Z}_{+},
\end{aligned}
$$

where $2<\beta_{1}<\beta_{2}$ and $\zeta(\cdot)$ denotes the Riemann zeta function. The matrix $\boldsymbol{P}$ in (5.38) can be regarded as the transition probability matrix of an irreducible reflected Markov additive process [1, Chapter XI, Section 2e]. It is easy to see that the stationary probability vector $\varpi$ of $\boldsymbol{A}=\sum_{k=-\infty}^{\infty} \boldsymbol{A}(k)$ is given by

$$
\varpi=\left(\begin{array}{ll}
\frac{1}{2} & \frac{1}{2}
\end{array}\right)
$$

For convenience, let

$$
a_{1}(k)=\left\{\begin{array}{ll}
2^{k-1}, & k \leq-1, \\
\frac{1}{2} \frac{(k+1)^{-\beta_{1}}}{\zeta\left(\beta_{1}\right)}, & k \in \mathbb{Z}_{+},
\end{array} \quad a_{2}(k)= \begin{cases}2^{k-1}, & k \leq-1, \\
\frac{1}{2} \frac{(k+1)^{-\beta_{2}}}{\zeta\left(\beta_{2}\right)}, & k \in \mathbb{Z}_{+} .\end{cases}\right.
$$

It then follows from (5.39), (5.40) and (5.42) that

$$
\boldsymbol{A}(k) \boldsymbol{e}=\left(\begin{array}{c}
a_{1}(k) \\
a_{2}(k)
\end{array}\right), \quad k \in \mathbb{Z},
$$

and thus

$$
\sum_{k=-\infty}^{\infty} k \boldsymbol{A}(k) \boldsymbol{e}=\left(\begin{array}{l}
\bar{a}_{1} \\
\bar{a}_{2}
\end{array}\right)
$$

where

$$
\begin{aligned}
& \bar{a}_{1}:=\sum_{k=-\infty}^{\infty} k a_{1}(k)=\frac{1}{2}\left(\frac{\zeta\left(\beta_{1}-1\right)}{\zeta\left(\beta_{1}\right)}-3\right), \\
& \bar{a}_{2}:=\sum_{k=-\infty}^{\infty} k a_{2}(k)=\frac{1}{2}\left(\frac{\zeta\left(\beta_{2}-1\right)}{\zeta\left(\beta_{2}\right)}-3\right) .
\end{aligned}
$$


Substituting (5.41) and (5.43) into (5.2) and using (5.44) and (5.45) yield

$$
\sigma=\frac{1}{2}\left(\bar{a}_{1}+\bar{a}_{2}\right)=\frac{1}{4}\left(\frac{\zeta\left(\beta_{1}-1\right)}{\zeta\left(\beta_{1}\right)}+\frac{\zeta\left(\beta_{2}-1\right)}{\zeta\left(\beta_{2}\right)}-6\right) .
$$

Note here that $1<\zeta\left(s_{2}\right)<\zeta\left(s_{1}\right)<\pi^{2} / 6$ for $2<s_{1}<s_{2}$, which leads to

$$
1<\frac{\zeta\left(\beta_{1}-1\right)}{\zeta\left(\beta_{1}\right)}<\frac{\pi^{2}}{6}<\frac{5}{3}, \quad 1<\frac{\zeta\left(\beta_{2}-1\right)}{\zeta\left(\beta_{2}\right)}<\frac{\pi^{2}}{6}<\frac{5}{3} .
$$

Therefore, from (5.44) and (5.45), we have

$$
\bar{a}_{1}<0, \quad \bar{a}_{2}<0 .
$$

Applying these two inequalities to (5.46), we obtain $\sigma<0$. i.e., Assumption 5.2 holds. As a result, $\boldsymbol{P}$ in (5.38) has the unique stationary probability vector $\boldsymbol{\pi}$.

Recall that Theorem 5.1 holds under all the conditions of Lemma 5.4. Therefore, to fulfill the conditions, we determine parameters $N \in \mathbb{N}, M \in \mathbb{N}, \kappa>0, \varepsilon \in(0,1), \delta_{0}>0, K_{0} \in$ $\{L, L+1, \ldots\}$ and $K \in\left\{K_{0}, K_{0}+1, \ldots\right\}$.

We begin with $N \in \mathbb{N}$ and $M \in \mathbb{N}$. In the present special case, the stochastic matrix $\boldsymbol{P}_{1}$ in (5.3) is expressed as

$$
\boldsymbol{P}_{1}=\left(\begin{array}{ccccc}
\underline{\boldsymbol{A}}(0) & \boldsymbol{A}(1) & \boldsymbol{A}(2) & \boldsymbol{A}(3) & \cdots \\
\underline{\boldsymbol{A}}(-1) & \boldsymbol{A}(0) & \boldsymbol{A}(1) & \boldsymbol{A}(2) & \ldots \\
\boldsymbol{O} & \underline{\boldsymbol{A}}(-1) & \boldsymbol{A}(0) & \boldsymbol{A}(1) & \cdots \\
\boldsymbol{O} & \boldsymbol{O} & \underline{\boldsymbol{A}}(-1) & \boldsymbol{A}(0) & \ldots \\
\vdots & \vdots & \vdots & \vdots & \ddots
\end{array}\right)
$$

Note here that (5.4) yields

$$
\boldsymbol{A}_{1}(-1)=\underline{\boldsymbol{A}}(-1)=\sum_{k=-\infty}^{-1} \boldsymbol{A}(k), \quad \boldsymbol{A}_{1}(k)=\boldsymbol{A}(k), \quad k \in \mathbb{Z}_{+}
$$

Thus, we have

$$
\sum_{k=-1}^{\infty} k \boldsymbol{A}_{1}(k) \boldsymbol{e}=-\underline{\boldsymbol{A}}(-1) \boldsymbol{e}+\sum_{k=1}^{\infty} k \boldsymbol{A}(k) \boldsymbol{e}=\left(\begin{array}{l}
\frac{1}{2}\left(\frac{\zeta\left(\beta_{1}-1\right)}{\zeta\left(\beta_{1}\right)}-2\right) \\
\frac{1}{2}\left(\frac{\zeta\left(\beta_{2}-1\right)}{\zeta\left(\beta_{2}\right)}-2\right)
\end{array}\right)<\mathbf{0}(5.50)
$$

where the second equality follows from (5.39) and (5.40), and where the last inequality follows from (5.47). Furthermore, applying (5.41) and (5.50) to (5.5) with $N=1$, we obtain

$$
\sigma_{1}=\frac{1}{4}\left(\frac{\zeta\left(\beta_{1}-1\right)}{\zeta\left(\beta_{1}\right)}+\frac{\zeta\left(\beta_{2}-1\right)}{\zeta\left(\beta_{2}\right)}-4\right)<0
$$


The inequalities (5.50) and (5.51) imply that (5.6) and (5.7) hold for $N=M_{0}=1$. To proceed, we fix $N=M=M_{0}=1$ and thus $L=M N=1$.

We then consider $\kappa>0$ and $\varepsilon \in(0,1)$. Using (5.49), we can reduce (5.20) to

$$
-(1-\varepsilon) \underline{\boldsymbol{A}}(-1) \boldsymbol{e}+(1+\varepsilon) \sum_{\ell=1}^{\infty} \ell \boldsymbol{A}(\ell) \boldsymbol{e} \leq-2 \kappa \boldsymbol{e} .
$$

Substituting (5.39) and (5.40) into the above inequality, we have

$$
\left(\begin{array}{c}
\frac{1+\varepsilon}{2} \frac{\zeta\left(\beta_{1}-1\right)}{\zeta\left(\beta_{1}\right)}-1 \\
\frac{1+\varepsilon}{2} \frac{\zeta\left(\beta_{2}-1\right)}{\zeta\left(\beta_{2}\right)}-1
\end{array}\right) \leq-2 \kappa\left(\begin{array}{l}
1 \\
1
\end{array}\right) .
$$

Since the Riemann zeta function $\zeta(s)$ is log-convex for $s>1$ (see, e.g., [4]),

$$
\begin{aligned}
\frac{\mathrm{d}}{\mathrm{d} s}\left(\frac{\zeta(s-1)}{\zeta(s)}\right) & =\frac{\zeta(s-1)}{\zeta(s)}\left(\frac{\zeta^{\prime}(s-1)}{\zeta(s-1)}-\frac{\zeta^{\prime}(s)}{\zeta(s)}\right) \\
& =\frac{\zeta(s-1)}{\zeta(s)}\left\{\frac{\mathrm{d}}{\mathrm{d} s} \log \zeta(s-1)-\frac{\mathrm{d}}{\mathrm{d} s} \log \zeta(s)\right\} \leq 0
\end{aligned}
$$

which leads to

$$
\frac{\zeta\left(\beta_{2}-1\right)}{\zeta\left(\beta_{2}\right)} \leq \frac{\zeta\left(\beta_{1}-1\right)}{\zeta\left(\beta_{1}\right)} .
$$

The inequalities (5.52) and (5.53) imply that (5.52) holds if

$$
\frac{1+\varepsilon}{2} \frac{\zeta\left(\beta_{1}-1\right)}{\zeta\left(\beta_{1}\right)}-1=-2 \kappa
$$

Therefore, we fix $\kappa>0$ and $\varepsilon \in(0,1)$ such that

$$
\begin{aligned}
& \kappa=\frac{1}{4}\left(1-\frac{\zeta\left(\beta_{1}-1\right)}{2 \zeta\left(\beta_{1}\right)}\right)>\frac{1}{24}, \\
& \varepsilon=\frac{1}{2}\left(\frac{2 \zeta\left(\beta_{1}\right)}{\zeta\left(\beta_{1}-1\right)}-1\right) \in\left(\frac{1}{10}, \frac{1}{2}\right),
\end{aligned}
$$

which satisfy (5.54) and thus (5.52).

Next, we determine $\delta_{0}>0, K_{0} \in\{L, L+1, \ldots\}$ (with $L=1$ ) and the function $V$ in Assumption 5.3. From (5.40), we have

$$
\lim _{k \rightarrow \infty} \frac{\boldsymbol{A}(k)}{k^{-\beta_{1}}}=\left(\begin{array}{cc}
0 & \frac{1}{2 \zeta\left(\beta_{1}\right)} \\
0 & 0
\end{array}\right),
$$

which corresponds to the case discussed in Appendix A.2. Thus, we fix $\alpha=0, \beta_{0} \in\left(1, \beta_{1}-1\right)$ arbitrarily and

$$
V(x)=\left(x+x_{0}\right)^{\beta_{0}}, \quad x \geq 0,
$$


with $x_{0}>0$. It then follows that Assumption 5.3 holds for $\alpha=0$ (see Appendix A.2). It also follows from (5.57), $\alpha=0$ and $L=1$ that (5.16) and (5.17) are reduced to

$$
\begin{array}{rlrl}
\frac{\left(\delta_{0}+1\right) k+x_{0}}{k+x_{0}} & \leq(1+\varepsilon)^{1 /\left(\beta_{0}-1\right)}, & & k \geq K_{0}+1, \\
\frac{k+x_{0}-1}{k+x_{0}} \geq(1-\varepsilon)^{1 /\left(\beta_{0}-1\right)}, & & k \geq K_{0}+1 .
\end{array}
$$

Note that

$$
\sup _{k \geq 0} \frac{\left(\delta_{0}+1\right) k+x_{0}}{k+x_{0}}=\delta_{0}+1, \quad \inf _{k \geq 0} \frac{k+x_{0}-1}{k+x_{0}}=1-\frac{1}{x_{0}} .
$$

Thus, we fix $K_{0} \in \mathbb{N}, \delta_{0}>0$ and $x_{0}>0$ such that

$$
\begin{aligned}
& K_{0}=1, \quad \delta_{0}=(1+\varepsilon)^{1 /\left(\beta_{0}-1\right)}-1, \\
& x_{0}=\frac{1}{1-(1-\varepsilon)^{1 /\left(\beta_{0}-1\right)}}
\end{aligned}
$$

where $\varepsilon$ is given in (5.56). It is easy to see that (5.58) and (5.59) hold.

Finally, we discuss the remaining parameter $K \in\left\{K_{0}, K_{0}+1, \ldots\right\}$ (with $K_{0}=1$ ). Let $\rho$ denote

$$
\rho=\max \left(1+1 / \delta_{0}, x_{0}\right)
$$

Let $C_{1}$ and $C_{2}$ denote

$$
C_{1}=\frac{\rho^{\beta_{0}} \delta_{0}^{-\beta_{1}+\beta_{0}+1}}{2 \beta_{0}\left(\beta_{1}-\beta_{0}-1\right) \zeta\left(\beta_{1}\right)}, \quad C_{2}=\frac{\rho^{\beta_{0}} \delta_{0}^{-\beta_{2}+\beta_{0}+1}}{2 \beta_{0}\left(\beta_{2}-\beta_{0}-1\right) \zeta\left(\beta_{2}\right)},
$$

respectively. Furthermore, fix

$$
K=\max \left(\left\lfloor\frac{C_{1}}{\kappa}\right\rfloor^{1 /\left(\beta_{1}-2\right)},\left\lfloor\frac{C_{2}}{\kappa}\right\rfloor^{1 /\left(\beta_{2}-2\right)}\right) .
$$

It then holds that

$$
\frac{1}{V^{\prime}(k)} \sum_{\ell=\left\lfloor\delta_{0} k\right\rfloor+1}^{\infty} V(k+\ell) \boldsymbol{A}(\ell) \boldsymbol{e} \leq \kappa \boldsymbol{e} \quad \text { for all } k \geq K+1
$$

which is proved in Appendix B. Inequality (5.64) together with (5.49) implies that (5.21) holds for $M=N=1, \alpha=0$ and $K$ given in (5.63).

We have confirmed that all the conditions of Lemma 5.4 hold for $N=M=1$. Therefore, Lemma 5.4 implies that

$$
\sum_{\ell=0}^{\infty} \boldsymbol{P}_{1}(k ; \ell) V(\ell) \boldsymbol{e}-V(k) \boldsymbol{e} \leq-\kappa V^{\prime}(k) \boldsymbol{e}, \quad k \geq K+1 .
$$

We now fix $\boldsymbol{v} \in \mathrm{BI}_{d}$ such that

$$
\boldsymbol{v}(k)=V(k) \boldsymbol{e}=\left(k+x_{0}\right)^{\beta_{0}} \boldsymbol{e}, \quad k \in \mathbb{Z}_{+},
$$


where $x_{0}$ is given in (5.60). We also fix $\phi$ as in (5.28). Thus, (5.29) holds. Applying (5.29) and (5.66) to (5.65) yields

$$
\sum_{\ell=0}^{\infty} \boldsymbol{P}_{1}(k ; \ell) \boldsymbol{v}(\ell)-\boldsymbol{v}(k) \leq-\phi \circ \boldsymbol{v}(k), \quad k \geq K+1 .
$$

Consequently, we can establish the drift conditions (5.33) and (5.34) with $N=M=1$ once we find an upper bound be for $\left\{\sum_{\ell=0}^{\infty} \boldsymbol{P}_{1}(k ; \ell) V(\ell) \boldsymbol{e}-V(k) \boldsymbol{e}+\kappa V^{\prime}(k) \boldsymbol{e} ; k=0,1, \ldots, K\right\}$.

We specify the parameter $b$. It follows from (5.39), (5.40) and (5.48) that

$$
\begin{aligned}
\sum_{\ell=0}^{\infty} & \boldsymbol{P}_{1}(0 ; \ell) V(\ell) \boldsymbol{e}-V(0) \boldsymbol{e}+\kappa V^{\prime}(0) \boldsymbol{e} \\
& =\underline{\boldsymbol{A}}(0) V(0) \boldsymbol{e}+\sum_{\ell=1}^{\infty} \boldsymbol{A}(\ell) V(\ell) \boldsymbol{e}-V(0) \boldsymbol{e}+\kappa V^{\prime}(0) \boldsymbol{e} \\
& \leq \sum_{\ell=1}^{\infty} \boldsymbol{A}(\ell) V(\ell) \boldsymbol{e}+\kappa V^{\prime}(0) \boldsymbol{e},
\end{aligned}
$$

where the inequality is due to $\underline{\boldsymbol{A}}(0) \boldsymbol{e} \leq \boldsymbol{e}$. Similarly, for $k=1,2, \ldots, K$,

$$
\begin{aligned}
& \sum_{\ell=0}^{\infty} \boldsymbol{P}_{1}(k ; \ell) V(\ell) \boldsymbol{e}-V(k) \boldsymbol{e}+\kappa V^{\prime}(k) \boldsymbol{e} \\
& \quad=\underline{\boldsymbol{A}}(-1) V(k-1) \boldsymbol{e}+\sum_{\ell=0}^{\infty} \boldsymbol{A}(\ell) V(k+\ell) \boldsymbol{e}-V(k) \boldsymbol{e}+\kappa V^{\prime}(k) \boldsymbol{e} \\
& \quad \leq \sum_{\ell=0}^{\infty} \boldsymbol{A}(\ell) V(k+\ell) \boldsymbol{e}+\kappa V^{\prime}(k) \boldsymbol{e},
\end{aligned}
$$

where the inequality holds because $\underline{\boldsymbol{A}}(-1) \boldsymbol{e} \leq \boldsymbol{e}$ and $V(k-1)<V(k)$ for all $k \in \mathbb{N}$. Since $V$ and $V^{\prime}$ is nondecreasing, we have, from (5.68) and (5.69),

$$
\begin{aligned}
& \sum_{\ell=0}^{\infty} \boldsymbol{P}_{1}(k ; \ell) V(\ell) \boldsymbol{e}-V(k) \boldsymbol{e}+\kappa V^{\prime}(k) \boldsymbol{e} \\
& \quad \leq \sum_{\ell=0}^{\infty} \boldsymbol{A}(\ell) V(K+\ell) \boldsymbol{e}+\kappa V^{\prime}(K) \boldsymbol{e}, \quad k=0,1, \ldots, K
\end{aligned}
$$

Applying (5.40) and (5.57) to the right hand side of the above inequality, we obtain

$$
\sum_{\ell=0}^{\infty} \boldsymbol{P}_{1}(k ; \ell) V(\ell) \boldsymbol{e}-V(k) \boldsymbol{e}+\kappa V^{\prime}(k) \boldsymbol{e} \leq b \boldsymbol{e}, \quad k=0,1, \ldots, K,
$$

where

$$
\begin{aligned}
b= & \max \left(\frac{1}{2 \zeta\left(\beta_{1}\right)} \sum_{\ell=0}^{\infty} \frac{\left(K+\ell+x_{0}\right)^{\beta_{0}}}{(\ell+1)^{\beta_{1}}}, \frac{1}{2 \zeta\left(\beta_{2}\right)} \sum_{\ell=0}^{\infty} \frac{\left(K+\ell+x_{0}\right)^{\beta_{0}}}{(\ell+1)^{\beta_{2}}}\right) \\
& +\kappa \beta_{0}\left(k+x_{0}\right)^{\beta_{0}-1} .
\end{aligned}
$$


Substituting (5.29) and (5.66) into (5.70) results in

$$
\sum_{\ell=0}^{\infty} \boldsymbol{P}_{1}(k ; \ell) \boldsymbol{v}(\ell)-\boldsymbol{v}(k)+\phi \circ \boldsymbol{v}(k) \leq b \boldsymbol{e}, \quad k=0,1, \ldots, K .
$$

Combining (5.72) with (5.67) leads to

$$
\boldsymbol{P}_{1} \boldsymbol{v} \leq \boldsymbol{v}-\phi \circ \boldsymbol{v}+b \mathbf{1}_{K}
$$

Recall here that $\boldsymbol{P} \prec_{d} \boldsymbol{P}_{1} \in \mathrm{BM}_{d}$ and $\boldsymbol{v} \in \mathrm{BI}_{d}$. Thus, $\boldsymbol{P} \boldsymbol{v} \leq \boldsymbol{P}_{1} \boldsymbol{v}$ (due to (5.9) with $N=M=$ 1). This inequality and (5.73) yield the drift condition on $P$ :

$$
\boldsymbol{P} \boldsymbol{v} \leq \boldsymbol{v}-\phi \circ \boldsymbol{v}+b \mathbf{1}_{K}
$$

In addition, $\boldsymbol{P}(k ; 0) \boldsymbol{e}=\underline{\boldsymbol{A}}(-k) \boldsymbol{e}>\boldsymbol{0}$ for all $k \in \mathbb{N}$, which follows from (5.38), (5.39) and (5.40).

We have shown that all the conditions of Theorem 5.1 are satisfied. We now fix

$$
B=2^{K} b
$$

where $b$ is given in (5.71). It then follows from (

$$
B \boldsymbol{P}(K ; 0) \boldsymbol{e}=2^{K} b \underline{\boldsymbol{A}}(-K) \boldsymbol{e}=b \boldsymbol{e} .
$$

As a result, using Theorem 5.1, we can derive an error bound for the special case considered here.

In what follows, we present the components of the error bound. Since $V^{-1}(t)=t^{1 / \beta_{0}}-x_{0}$ for $t \geq x_{0}^{\beta_{0}}$, the function $\phi$ in (5.28) is expressed as

$$
\phi(t)=\kappa \beta_{0} t^{1-1 / \beta_{0}}, \quad t \geq 1
$$

Therefore, the function $H_{\phi}$ in (3.4) is given by

$$
H_{\phi}(x)=\int_{1}^{x} \frac{y^{-1+1 / \beta_{0}}}{\kappa \beta_{0}} \mathrm{~d} y=\kappa^{-1}\left(x^{1 / \beta_{0}}-1\right), \quad x \geq 1 .
$$

Substituting (5.75) and (5.76) into (3.5), we have

$$
r_{\phi}(x)=\kappa \beta_{0}(\kappa x+1)^{\beta_{0}-1}, \quad x \geq 0 .
$$

Furthermore, applying (5.74) and (5.75) to (4.5), we obtain

$$
c_{\phi, B}(x)=\breve{c} x, \quad x \geq 0,
$$

where

$$
\breve{c}=\left(2^{K} b+1\right)^{-1+1 / \beta_{0}} .
$$


Combining (5.77) and (5.78) yields

$$
r_{\phi} \circ c_{\phi, B}(x)=\kappa \beta_{0}(\kappa \breve{c} x+1)^{\beta_{0}-1}, \quad x \geq 0 .
$$

Consequently, letting $M=1$ and $d=2$ in (5.36) and using (5.57), (5.74), (5.78) and (5.80), we obtain

$$
\begin{aligned}
\left\|_{(n)} \boldsymbol{\pi}_{n}-\boldsymbol{\pi}\right\| \leq & \frac{8 \breve{c}^{-1}}{\kappa \beta_{0}\{\kappa \breve{c}(m-1)+1\}^{\beta_{0}-1}}\left\{\left(1+x_{0}\right)^{\beta_{0}}+2^{K} b\right\} \\
& +\frac{4 m b}{\kappa \beta_{0}\left(n+x_{0}\right)^{\beta_{0}-1}} \quad \text { for all } m, n \in \mathbb{N},
\end{aligned}
$$

where $\kappa, x_{0}, K, b$ and $\breve{c}$ are given by (5.55), (5.60), (5.63), (5.71) and (5.79), respectively.

Finally, using the obtained bound (5.81), we determine a truncation parameter $n \in \mathbb{N}$ such that $\left\|_{(n)} \boldsymbol{\pi}_{n}-\boldsymbol{\pi}\right\|$ is within a given tolerance $\mathcal{E} \in(0,2)$, i.e.,

$$
\left\|_{(n)} \boldsymbol{\pi}_{n}-\boldsymbol{\pi}\right\| \leq \mathcal{E}
$$

Let $m_{0}$ and $n_{0}$ denote

$$
\begin{aligned}
& m_{0}=\min \left\{m \in \mathbb{N} ; \frac{8 \breve{c}^{-1}}{\kappa \beta_{0}\{\kappa \breve{c}(m-1)+1\}^{\beta_{0}-1}}\left\{\left(1+x_{0}\right)^{\beta_{0}}+2^{K} b\right\} \leq \frac{\mathcal{E}}{2}\right\}, \\
& n_{0}=\min \left\{n \in \mathbb{N} ; \frac{4 m_{0} b}{\kappa \beta_{0}\left(n+x_{0}\right)^{\beta_{0}-1}} \leq \frac{\mathcal{E}}{2}\right\}
\end{aligned}
$$

respectively. We then have

$$
\begin{aligned}
& m_{0}=\left\lceil\frac{1}{\kappa \breve{c}}\left(\left[\frac{16 \breve{c}^{-1}}{\kappa \beta_{0} \mathcal{E}}\left\{\left(1+x_{0}\right)^{\beta_{0}}+2^{K} b\right\}\right]^{1 /\left(\beta_{0}-1\right)}-1\right)\right]+1, \\
& n_{0}=\max \left(1,\left[\left(\frac{8 m_{0} b}{\kappa \beta_{0} \mathcal{E}}\right)^{1 /\left(\beta_{0}-1\right)}-x_{0}\right]\right) .
\end{aligned}
$$

Substituting $m_{0}$ in (5.83) and $n_{0}$ in (5.84) into (5.81) yields (5.82).

\section{Concluding remarks}

This paper studied the estimation of the total variation distance between the stationary probability vectors of a discrete-time block-structured Markov chain and its LC-block-augmented truncation. The main contribution of this paper is to present a total-variation-distance error bound for the stationary probability vector of the LC-block-augmented truncation under the assumption that the original Markov chain is block monotone and satisfies the subgeometric drift condition proposed in [3]. This paper is complementary to the author's previous study [18], which considered discrete-time block-monotone Markov chains satisfying the geometric drift condition. The author [20] also considered continuous-time block-monotone Markov chains 
with exponential ergodicity and derived a total-variation-distance error bound for the stationary probability vector of the LC-block-augmented truncation. The present study and the author's previous ones [18, 20] depend on the notion of block monotonicity.

Recently, without block monotonicity (including monotonicity), the author [21] established computable upper bounds for the absolute difference between the time-averaged functionals of a continuous-time block-structured Markov chain and its LC-block-augmented truncation under the assumption that the original Markov chain satisfies the $f$-modulated drift condition (see [21, Condition 1,1]). The $f$-modulated drift condition includes the geometric (or exponential in continuous time) drift condition and the subgeometric (or subexponential in continuous time) drift condition proposed in [3] as special cases. Therefore, the error bounds in [21] are widely applicable, though they are more computationally costly than those in this paper and [18, 20] with block monotonicity.

\section{A Examples of function $V$ in Assumption 5.3}

We begin with the following lemma.

Lemma A.1 Equation (5.10) holds if there exists some $\alpha \in[0,1)$ such that at least one of (A.1) and (A.2) below is true for any $\delta>0$.

$$
\begin{aligned}
& \lim _{k \rightarrow \infty} \frac{V(k)}{V^{\prime}(k)} \sum_{\ell=\left\lfloor\delta k^{1-\alpha}\right\rfloor+1}^{\infty} V(\ell) \boldsymbol{A}_{N}^{* M}(\ell) \boldsymbol{e}=\mathbf{0}, \\
& \limsup _{\ell \rightarrow \infty} \frac{V(\delta \ell)}{V(\ell)}<\infty \quad \text { and } \quad \sum_{\ell=0}^{\infty} V\left(\ell^{1 /(1-\alpha)}\right) \boldsymbol{A}_{N}^{* M}(\ell) \boldsymbol{e} \text { is finite, }
\end{aligned}
$$

where $V:[0, \infty) \rightarrow[1, \infty)$ is an increasing, convex and log-concave function that satisfies conditions (i)-(v) of Assumption 5.3

Proof. Since $V$ is $\log$-concave and $\log V(0) \geq 0$, we have, for $x, y \geq 0$,

$$
\begin{aligned}
\log V(x)+\log V(y) \geq & \left(\frac{x}{x+y} \log V(x+y)+\frac{y}{x+y} \log V(0)\right) \\
& +\left(\frac{y}{x+y} \log V(x+y)+\frac{x}{x+y} \log V(0)\right) \\
\geq & \log V(x+y),
\end{aligned}
$$

and thus $V(x+y) \leq V(x) V(y)$ for $x, y \geq 0$. Using this inequality, we have

$$
\sum_{\ell=\left\lfloor\delta k^{1-\alpha}\right\rfloor+1}^{\infty} V(k+\ell) \boldsymbol{A}_{N}^{* M}(\ell) \boldsymbol{e} \leq V(k) \sum_{\ell=\left\lfloor\delta k^{1-\alpha}\right\rfloor+1}^{\infty} V(\ell) \boldsymbol{A}_{N}^{* M}(\ell) \boldsymbol{e} .
$$

Therefore, (A.1) implies (5.10). 
Next, we prove that (A.2) implies (5.10). To this end, we suppose that (A.2) holds. It then follows that, for any $c>0, \sum_{\ell=0}^{\infty} V\left(c \ell^{1 /(1-\alpha)}\right) \boldsymbol{A}_{N}^{* M}(\ell) \boldsymbol{e}$ is finite and thus

$$
\lim _{k \rightarrow \infty} \sum_{\ell=\left\lfloor\delta k^{1-\alpha}\right\rfloor+1}^{\infty} V\left(c \ell^{1 /(1-\alpha)}\right) \boldsymbol{A}_{N}^{* M}(\ell) \boldsymbol{e}=\mathbf{0} .
$$

In addition, since $V$ is increasing and convex,

$$
\liminf _{k \rightarrow \infty} V^{\prime}(k)>0
$$

and, for any $\delta>0$,

$$
\begin{aligned}
\sum_{\ell=\left\lfloor\delta k^{1-\alpha}\right\rfloor+1}^{\infty} V(k+\ell) \boldsymbol{A}_{N}^{* M}(\ell) \boldsymbol{e} & \leq \sum_{\ell=\left\lfloor\delta k^{1-\alpha}\right\rfloor+1}^{\infty} V\left(\ell+(\ell / \delta)^{1 /(1-\alpha)}\right) \boldsymbol{A}_{N}^{* M}(\ell) \boldsymbol{e} \\
& \leq \sum_{\ell=\left\lfloor\delta k^{1-\alpha}\right\rfloor+1}^{\infty} V\left(c_{\delta} \ell^{1 /(1-\alpha)}\right) \boldsymbol{A}_{N}^{* M}(\ell) \boldsymbol{e} \quad k \in \mathbb{Z}_{+},
\end{aligned}
$$

where $c_{\delta} \geq 1+\delta^{-1 /(1-\alpha)}$. Combining (A.3), (A.4) and (A.5), we have (5.10).

Using Lemma A.1, we present the typical examples of the function $V$ satisfying Assumption 5.3 , by considering the three cases:

(a) $\boldsymbol{A}(k) \asymp g_{1}(k):=\exp \left\{-c k^{\alpha}\right\}$ for some $c>0$ and $0<\alpha<1$;

(b) $\boldsymbol{A}(k) \asymp g_{2}(k):=k^{-\beta}$ for some $\beta>2$; and

(c) $\boldsymbol{A}(k) \asymp g_{3}(k):=k^{-2}\{\log (k+1)\}^{-\gamma}$ for some $\gamma>1$,

where we write $\boldsymbol{H}(x) \asymp g(x)$ if $\boldsymbol{H}$ is a nonnegative matrix-valued function such that both $\liminf _{x \rightarrow \infty} \boldsymbol{H}(x) / g(x)$ and $\limsup _{x \rightarrow \infty} \boldsymbol{H}(x) / g(x)$ are finite and not equal to the zero matrix for a scalar-valued function $g$ that is eventually nonnegative. For $i=1,2,3$, it follows from $\boldsymbol{A}(k) \asymp g_{i}(k)$ and (5.4) that $\boldsymbol{A}_{N}(k) \asymp g_{i}(k)$. Thus, $\boldsymbol{A}_{N}^{* M}(k) \asymp g_{i}(k)$ for $i=1,2,3$, which can be readily proved by using the extensions of [9, Proposition A.2.6] to the upper and lower limits.

We will see later, from the examples of the function $V$, that the decay of the error bound (5.36) is moderately exponential (i.e., heavy-tailed Weibull-like) in Case (a); polynomial in Case (b); and logarithmic in Case (c), as the truncation parameter $n$ increases. Therefore, Cases (a), (b) and (c) are called moderately exponential case, polynomial case, and logarithmic case, respectively.

\section{A.1 Moderately exponential case}

We suppose that $\boldsymbol{A}(k) \asymp g_{1}(k)$, i.e., $\boldsymbol{A}(k) \asymp \exp \left\{-c k^{\alpha}\right\}$ for some $c>0$ and $0<\alpha<1$. We then fix $V$ such that

$$
V(x)=\exp \left\{c_{0}\left(x+x_{0}\right)^{\alpha}\right\}, \quad x \geq 0
$$


where $0<c_{0}<c$ and $x_{0} \geq 1 /\left(\alpha c_{0}\right)^{1 / \alpha}$. Clearly, $V$ is increasing and log-concave, and conditions (i) and (ii) of Assumption 5.3 are satisfied. In what follows, we confirm that the remaining conditions of Assumption 5.3 are satisfied.

From (A.6), we have

$$
\begin{aligned}
V^{\prime}(x) & =\alpha c_{0}\left(x+x_{0}\right)^{\alpha-1} \exp \left\{c_{0}\left(x+x_{0}\right)^{\alpha}\right\}>0, \quad x>0, \\
V^{\prime \prime}(x) & =\alpha c_{0}\left(x+x_{0}\right)^{\alpha-2} \exp \left\{c_{0}\left(x+x_{0}\right)^{\alpha}\right\}\left\{\alpha c_{0}\left(x+x_{0}\right)^{\alpha}-(1-\alpha)\right\}, \quad x>0(\text { A.8. })
\end{aligned}
$$

which yield

$$
\begin{aligned}
\frac{V^{\prime}(x)}{V(x)} & =\alpha c_{0}\left(x+x_{0}\right)^{\alpha-1}, \quad x>0, \\
\frac{V^{\prime \prime}(x)}{V^{\prime}(x)} & =\left(x+x_{0}\right)^{-1}\left\{\alpha c_{0}\left(x+x_{0}\right)^{\alpha}-(1-\alpha)\right\}, \quad x>0 .
\end{aligned}
$$

Equation (A.7) implies that condition (iii) of Assumption 5.3 holds and that

$$
\begin{aligned}
\lim _{\delta \downarrow 0} \lim _{x \rightarrow \infty} \frac{V^{\prime}\left(x+\delta x^{1-\alpha}\right)}{V^{\prime}(x)}= & \lim _{\delta \downarrow 0} \lim _{x \rightarrow \infty}\left(1+\frac{\delta x^{1-\alpha}}{x+x_{0}}\right)^{\alpha-1} \\
& \times \exp \left\{c_{0}\left(x+x_{0}\right)^{\alpha}\left[\left(1+\frac{\delta x^{1-\alpha}}{x+x_{0}}\right)^{\alpha}-1\right]\right\} \\
= & \lim _{\delta \downarrow 0} \lim _{x \rightarrow \infty} \exp \left\{c_{0}\left(x+x_{0}\right)^{\alpha} \frac{\alpha \delta x^{1-\alpha}}{x+x_{0}}\right\} \\
= & \lim _{\delta \downarrow 0} \lim _{x \rightarrow \infty} \exp \left\{\alpha c_{0} \delta\left(\frac{x}{x+x_{0}}\right)^{1-\alpha}\right\} \\
= & \lim _{\delta \downarrow 0} \exp \left\{\alpha c_{0} \delta\right\}=1,
\end{aligned}
$$

which shows that condition (v) of Assumption 5.3 holds. In addition, since $x_{0} \geq 1 /\left(\alpha c_{0}\right)^{1 / \alpha}>$ $\left\{(1-\alpha) /\left(\alpha c_{0}\right)\right\}^{1 / \alpha}$, it follows from (A.8) and (A.10) that, for $x>0, V^{\prime \prime}(x)>0$ and $V^{\prime \prime}(x) / V^{\prime}(x)$ is nonincreasing, i.e., condition (iv) of Assumption 5.3 is satisfied.

Finally, we confirm that (5.10) holds. It follows from (A.6), (A.9) and $\boldsymbol{A}_{N}^{* M}(k) \asymp \exp \left\{-c k^{\alpha}\right\}$ that there exists some finite $C>0$ such that, for all sufficiently large $k$,

$$
\frac{V(k)}{V^{\prime}(k)} \sum_{\ell=\left\lfloor\delta k^{1-\alpha}\right\rfloor+1}^{\infty} V(\ell) \boldsymbol{A}_{N}^{* M}(\ell) \boldsymbol{e} \leq C\left(k+x_{0}\right)^{1-\alpha} \sum_{\ell=\left\lfloor\delta k^{1-\alpha}\right\rfloor+1}^{\infty} \exp \left\{-\left(c-c_{0}\right) \ell^{\alpha}\right\} \boldsymbol{e}
$$

which implies that

$$
\lim _{k \rightarrow \infty} \frac{V(k)}{V^{\prime}(k)} \sum_{\ell=\left\lfloor\delta k^{1-\alpha}\right\rfloor+1}^{\infty} V(\ell) \boldsymbol{A}_{N}^{* M}(\ell) \boldsymbol{e}=\mathbf{0} .
$$

Combining this and Lemma A.1, we have (5.10). Consequently, the function $V$ given in (A.6) satisfies all the conditions of Assumption 5.3. 


\section{A.2 Polynomial case}

We suppose that $\boldsymbol{A}(k) \asymp g_{2}(k)=k^{-\beta}$ for some $\beta>2$, and fix $V$ such that

$$
V(x)=\left(x+x_{0}\right)^{\beta_{0}}, \quad x \geq 0,
$$

where $1<\beta_{0}<\beta-1$ and $x_{0}>0$. From (A.11, we have

$$
\begin{aligned}
V^{\prime}(x) & =\beta_{0}\left(x+x_{0}\right)^{\beta_{0}-1}>0, \quad x>0, \\
V^{\prime \prime}(x) & =\beta_{0}\left(\beta_{0}-1\right)\left(x+x_{0}\right)^{\beta_{0}-2}>0, \quad x>0 .
\end{aligned}
$$

Clearly, $V$ is increasing, convex and log-concave, and conditions (i)-(iv) of Assumption 5.3 are satisfied. From (A.12), we also obtain

$$
\lim _{\delta \downarrow 0} \lim _{x \rightarrow \infty} \frac{V^{\prime}(x+\delta x)}{V^{\prime}(x)}=\lim _{\delta \downarrow 0}(1+\delta)^{\beta_{0}-1}=1,
$$

and thus condition (v) of Assumption 5.3 holds for $\alpha=0$. Furthermore, it follows from (A.11), $\alpha=0$ and $\boldsymbol{A}(k) \asymp k^{-\beta}$ that

$$
\begin{aligned}
& \lim _{k \rightarrow \infty} \frac{V(\delta k)}{V(k)}=\delta^{\beta_{0}} \quad \text { for any } \delta>0, \\
& V\left(k^{1 /(1-\alpha)}\right) \boldsymbol{A}_{N}^{* M}(k) \asymp k^{\beta_{0}-\beta} .
\end{aligned}
$$

These equations, together with $\beta_{0}-\beta<-1$, imply (A.2). Therefore, Lemma A.1 shows that (5.10) holds for $\alpha=0$. We have confirmed that the function $V$ given in (A.11) satisfies all the conditions of Assumption 5.3.

\section{A.3 Logarithmic case}

We suppose that $\boldsymbol{A}(k) \asymp g_{3}(k)=k^{-2}\{\log (k+1)\}^{-\gamma}$ for some $\gamma>1$, and fix $V$ such that

$$
V(x)=\left(x+x_{0}\right)\left\{\log \left(x+x_{0}\right)\right\}^{\gamma_{0}}, \quad x \geq 0,
$$

where $0<\gamma_{0}<\gamma-1$ and $x_{0} \geq \mathrm{e}^{2}$. From (A.13), we have

$$
\begin{aligned}
V^{\prime}(x) & =\left\{\log \left(x+x_{0}\right)\right\}^{\gamma_{0}-1}\left[\log \left(x+x_{0}\right)+\gamma_{0}\right]>0, \quad x>0, \\
V^{\prime \prime}(x) & \left.=\gamma_{0}\left(x+x_{0}\right)^{-1}\left\{\log \left(x+x_{0}\right)\right\}^{\gamma_{0}-2}\left[\log \left(x+x_{0}\right)+\gamma_{0}-1\right]>0, \quad x>\text { (A. } 14\right)
\end{aligned}
$$

Therefore, $V$ is increasing, convex and log-concave and, conditions (i)-(iii) of Assumption 5.3 are satisfied.

In addition, (A.14) and (A.15) yield

$$
\frac{V^{\prime \prime}(x)}{V^{\prime}(x)}=\gamma_{0}\left(x+x_{0}\right)^{-1}\left[\left\{\log \left(x+x_{0}\right)\right\}^{-1} \frac{\log \left(x+x_{0}\right)+\gamma_{0}-1}{\log \left(x+x_{0}\right)+\gamma_{0}}\right], \quad x>0 .
$$


We now set $y=\log \left(x+x_{0}\right) \geq 2$ and denote by $F(y)$ the part in the square bracket in the right hand side of (A.16), i.e.,

$$
F(y)=\frac{y+\gamma_{0}-1}{y\left(y+\gamma_{0}\right)}, \quad y \geq 2
$$

We then have

$$
F^{\prime}(y)=-\frac{\left(y+\gamma_{0}-1\right)^{2}+\gamma_{0}-1}{\left\{y\left(y+\gamma_{0}\right)\right\}^{2}}<0 \quad \text { for all } y \geq 2
$$

Consequently, $V^{\prime \prime}(x) / V^{\prime}(x)$ is nonincreasing for all $x>0$, i.e., condition (iv) of Assumption 5.3 holds.

It remains to show that (5.10) and condition (v) of Assumption 5.3 hold. It follows from (A.14) that

$$
\lim _{x \rightarrow \infty} \frac{V^{\prime}(x+\delta x)}{V^{\prime}(x)}=1 \quad \text { for any } \delta>0
$$

which shows that condition (v) of Assumption 5.3 holds for $\alpha=0$. It also follows from (A.13), $\alpha=0$ and $\boldsymbol{A}_{N}^{* M}(k) \asymp k^{-2}\{\log (k+1)\}^{-\gamma}$ that

$$
\begin{aligned}
\lim _{k \rightarrow \infty} \frac{V(\delta k)}{V(k)} & =\delta \quad \text { for any } \delta>0, \\
V\left(k^{1 /(1-\alpha)}\right) \boldsymbol{A}_{N}^{* M}(k) & \asymp k^{-1}\{\log (k+1)\}^{\gamma_{0}-\gamma} .
\end{aligned}
$$

Combining these equations with $\gamma_{0}-\gamma<-1$, we have (A.2). Therefore, (5.10) holds for $\alpha=0$ (see Lemma A.1). As a result, the function $V$ given in A.13) satisfies all the conditions of Assumption 5.3 .

\section{B Proof of (5.64)}

We first note that A.5 holds for any $\alpha \in[0,1)$ and $\delta>0$. We then fix $M=N=1, \alpha=0$, $\delta=\delta_{0}$ and $c_{\delta_{0}}=\rho \geq 1+1 / \delta_{0}$, where $\rho$ is given in (5.61). It then follows from (A.5), (5.40), 
(5.49) and (5.57) that

$$
\begin{aligned}
& \frac{1}{V^{\prime}(k)} \sum_{\ell=\left\lfloor\delta_{0} k\right\rfloor+1}^{\infty} V(k+\ell) \boldsymbol{A}(\ell) \boldsymbol{e} \\
& \leq \frac{1}{V^{\prime}(k)} \sum_{\ell=\left\lfloor\delta_{0} k\right\rfloor+1}^{\infty} V(\rho \ell) \boldsymbol{A}(\ell) \boldsymbol{e} \\
& \leq \frac{\rho^{\beta_{0}}}{2 \beta_{0}\left(k+x_{0}\right)^{\beta_{0}-1}}\left(\sum_{\ell=\left\lfloor\delta_{0} k\right\rfloor+1}^{\infty} \frac{\left(\ell+x_{0} / \rho\right)^{\beta_{0}}(\ell+1)^{-\beta_{1}}}{\zeta\left(\beta_{1}\right)}\right) \\
& \leq \frac{\sum^{\beta_{0}}}{2 \beta_{0} k^{\beta_{0}-1}}\left(\sum_{\ell=\left\lfloor\delta_{0} k\right\rfloor+1}^{\infty} \frac{\left(\ell+x_{0} / \rho\right)^{\beta_{0}}(\ell+1)^{-\beta_{2}}}{\zeta\left(\beta_{2}\right)}\right), k \in \mathbb{N}, \\
& \left.\sum_{\ell=\left\lfloor\delta_{0} k\right\rfloor+1}^{\infty} \frac{(\ell+1)^{-\beta_{2}+\beta_{0}}}{\zeta\left(\beta_{2}\right)}\right)
\end{aligned}
$$

where the last inequality follows from $x_{0}>0$ and $x_{0} / \rho \leq 1$ (due to (5.61)). Note here that

$$
\sum_{\ell=\left\lfloor\delta_{0} k\right\rfloor+1}^{\infty}(\ell+1)^{-\beta} \leq \int_{\left\lfloor\delta_{0} k\right\rfloor}^{\infty}(x+1)^{-\beta} \mathrm{d} x \leq \frac{\left(\delta_{0} k\right)^{-\beta+1}}{\beta-1}, \quad \beta>1 .
$$

Applying this inequality to (B.1) yields, for $k \in \mathbb{N}$,

$$
\begin{aligned}
\frac{1}{V^{\prime}(k)} \sum_{\ell=\left\lfloor\delta_{0} k\right\rfloor+1}^{\infty} V(k+\ell) \boldsymbol{A}(\ell) \boldsymbol{e} & \leq \frac{\rho^{\beta_{0}}}{2 \beta_{0} k^{\beta_{0}-1}}\left(\begin{array}{c}
\frac{\left(\delta_{0} k\right)^{-\beta_{1}+\beta_{0}+1}}{\left(\beta_{1}-\beta_{0}-1\right) \zeta\left(\beta_{1}\right)} \\
\frac{\left(\delta_{0} k\right)^{-\beta_{2}+\beta_{0}+1}}{\left(\beta_{2}-\beta_{0}-1\right) \zeta\left(\beta_{2}\right)}
\end{array}\right) \\
& =\frac{\rho^{\beta_{0}}}{2 \beta_{0}}\left(\begin{array}{c}
\delta_{0}^{-\beta_{1}+\beta_{0}+1} \frac{k^{-\beta_{1}+2}}{\left(\beta_{1}-\beta_{0}-1\right) \zeta\left(\beta_{1}\right)} \\
\delta_{0}^{-\beta_{2}+\beta_{0}+1} \frac{k^{-\beta_{2}+2}}{\left(\beta_{2}-\beta_{0}-1\right) \zeta\left(\beta_{2}\right)}
\end{array}\right) \\
& =\left(\begin{array}{c}
C_{1} k^{-\beta_{1}+2} \\
C_{2} k^{-\beta_{2}+2}
\end{array}\right)
\end{aligned}
$$

where the last equality follows from (5.62). Finally, (B.2) and (5.63) imply that (5.64) holds for all $k \geq K+1$.

Acknowledgments. The author acknowledges stimulating discussions with Shusaku Sakaiya.

\section{References}

[1] S. Asmussen, Applied Probability and Queues, 2nd ed., Springer, New York, 2003. 
[2] P. BrÉmaud, Markov Chains: Gibbs Fields, Monte Carlo Simulation, and Queues, Springer, New York, 1999.

[3] R. Douc, G. ForT, AND E. Moulines, Practical drift conditions for subgeometric rates of convergence, Ann. Appl. Prob., 14 (2004), pp. 1353-1377.

[4] A. GuT, Some remarks on the Riemann zeta distribution, Preprint, U.U.D.M. Report 2005:6, ISSN 1101-3591, Department of Mathematics, Uppsala University, 2005.

[5] A. G. HART and R. L. TweEDIE, Convergence of Invariant Measures of Truncation Approximations to Markov Processes, Appl. Math., 3 (2012), 2205-2215.

[6] Q.-M. HE, Fundamentals of Matrix-Analytic Methods, Springer, New York, 2014.

[7] L. HeRVÉ AND J. LedouX, Approximating Markov chains and V-geometric ergodicity via weak perturbation theory, Stoch. Process. Appl., 124 (2014), pp. 613-638.

[8] S. F. JARNER and R. L. TWEEDIE, Necessary conditions for geometric and polynomial ergodicity of random-walk-type Markov chains, Bernoulli, 9 (2003), pp. 559-578.

[9] T. Kimura, H. Masuyama, And Y. TAKahashi, Subexponential asymptotics of the stationary distributions of GI/G/1-type Markov chain, Stoch. Models, 29 (2013), pp. 190239.

[10] H. LI AND Y. Q. ZHAO, Stochastic block-monotonicity in the approximation of the stationary distribution of infinite Markov chains, Stoch. Models, 16 (2000), pp. 313-333.

[11] Q.-L. LI AND Y. Q. ZHAO, Heavy-tailed asymptotics of stationary probability vectors of Markov chains of GI/G/l type, Adv. Appl. Prob., 37 (2005), pp. 482-509.

[12] Y. LIU, Augmented truncation approximations of discrete-time Markov chains, Oper. Res. Lett., 38 (2010), pp. 218-222.

[13] Y. LIU, Perturbation analysis for continuous-time Markov chains, Sci. China Math., 58 (2015), pp. 2633-2642.

[14] Y. MaO, Y. TAI, Y. Q. ZhaO, AND J. ZoU, Ergodicity for the GI/G/l-type Markov chain, J. Appl. Prob. Statist., 9 (2014), pp. 31-44.

[15] H. Masuyama, B. LiU, And T. TAKIne, Subexponential asymptotics of the BMAP/GI/I queue, J. Oper. Res. Soc. Jpn., 52 (2009), pp. 377-401.

[16] H. Masuyama, Subexponential asymptotics of the stationary distributions of $M / G / 1$-type Markov chains, Eur. J. Oper. Res., 213 (2011), pp. 509-516. 
[17] H. MASUyAma, Tail asymptotics for cumulative processes sampled at heavy-tailed random times with applications to queueing models in Markovian environments, J. Oper. Res. Soc. Jpn., 56 (2013), pp. 257-308.

[18] H. MASUYAMA, Error bounds for augmented truncations of discrete-time blockmonotone Markov chains under geometric drift conditions, Adv. Appl. Prob., 47 (2015), pp. 83-105.

[19] H. MASUYAma, A sufficient condition for the subexponential asymptotics of GI/G/1-type Markov chains with queueing applications, Ann. Oper. Res., 247 (2016), pp. 65-95, 2016.

[20] H. Masuyama, Continuous-time block-monotone Markov chains and their blockaugmented truncations, Lin. Alg. Appl., 514 (2017), pp. 105-150.

[21] H. MASUYAma, Error bounds for last-column-block-augmented truncations of blockstructured Markov chains, To appear in J. Oper. Res. Soc. Jpn., 60 (2017) (Preprint arXiv:1601.03489).

[22] S. P. Meyn And R. L. TweEdie, Markov Chains and Stochastic Stability, 2nd ed., Cambridge University Press, Cambridge, 2009.

[23] B. Sengupta, The semi-Markovian queue: theory and applications, Stoch. Models, 6 (1990), pp. 383-413.

[24] R. L. TweEDIE, Truncation approximations of invariant measures for Markov chains, J. Appl. Prob., 35 (1998), pp. 517-536.

[25] A. Zeifman, V. Korolev, Y. Satin, A. Korotysheva, and V. Bening, Perturbation bounds and truncations for a class of Markovian queues, Queueing Syst., 76 (2014), pp. 205-221.

[26] A. Zeifman And A. Korotysheva, Perturbation bounds for $\mathrm{M}_{t} / \mathrm{M}_{t} / N$ queue with catastrophes, Stoch. Models, 28, (2012), pp. 49-62.

[27] A. Zeifman, Y. Satin, V. Korolev, And S. Shorgin, On truncations for weakly ergodic inhomogeneous birth and death processes, Int. J. Appl. Math. Comput. Sci., 24 (2014), pp. 503-518. 